\title{
On the Choquet multiple criteria preference aggregation model: Theoretical and practical insights from a real-world application
}

\author{
M. Bottero a, V. Ferretti b, c, J. R. Figueira d, *, S. Greco e,f, B. Roy ${ }^{\text {g }}$ \\ a Department of Regional and Urban Studies and Planning, Politecnico di Torino, Italy \\ bepartment of Architecture, Built Environment and Construction Engineering (ABC), Politecnico di Milano, Italy \\ ${ }^{\mathrm{c}}$ Department of Management, London School of Economics and Political Science, London, United Kingdom \\ ${ }^{\mathrm{d}}$ CEG-IST, Instituto Superior Técnico, Universidade de Lisboa, Portugal \\ e Department of Economics and Business, University of Catania, Sicily, Italy \\ ${ }^{\mathrm{f}}$ Portsmouth Business School, Centre of Operations Research and Logistics (CORL), University of Portsmouth, Portsmouth, United Kingdom \\ g Université Paris-Dauphine, PSL Research University, CNRS (UMR 7243), LAMSADE, Paris, France
}

\section{ABSTRACT}

We consider the use of the Choquet integral for evaluating projects or actions in a real-world application starting from the case of the re-qualification of an abandoned quarry. Despite the Choquet integral being a very well-known preference model for which there is a rich and well developed theory, its application in a multiple criteria decision aiding perspective requires some specific methodological developments. This led us to work out and implement in practice two new procedures: a first procedure to build interval scales with the objective of assigning utility values on a common scale to the criteria performances, and a second one to construct a ratio scale for assigning numerical values to the capacities of the Choquet integral. This article discusses the strengths and weaknesses of the Choquet integral as appearing in the case study, proposing as well insights related to the interaction of the experts within a focus group.

\section{Introduction}

The starting point for this research is the work developed in a previous study (see Bottero, Ferretti, Figueira, Greco, \& Roy, 2015) in which we handled the interaction between some pairs of criteria within the context of an outranking method, namely ELECTRE III, properly generalized to this aim in Figueira, Greco, and Roy (2009). Interaction among criteria is a crucial aspect of decision aiding that is attracting more and more attention. That is why we took advantage of the opportunity we had (it should be underlined that this was a very exceptional situation) to work with the same experts as in the previous study, in order to evaluate the same five alternative re-qualification projects or actions for an abandoned quarry, by making use now of the multiple criteria preference aggregation Choquet integral (Choquet, 1953) model.

Indeed, the Choquet integral is a well-known technique for dealing with interaction among criteria (see e.g. Grabisch \& Labreuche, 2010; 2016). Differently from the usual weighted-sum, in which a weight is assigned to each criterion, the Choquet integral is based on the so-called capacities, also known as fuzzy

* Corresponding author at: CEG-IST, Instituto Superior Técnico, Universidade de Lisboa, Av. Rovisco Pais 1, 1049-001, Lisboa, Portugal.

E-mail address: figueira@tecnico.ulisboa.pt (J.R. Figueira). measures; it assigns an overall weight (hereafter, with some abuse of language, also called capacity), $\mu(T)$, to each subset, $T$, of the considered set of criteria, $G$. In reality, the capacity or weight, $\mu(T)$, can be interpreted as the value assigned to a dummy project corresponding to an action having completely satisfactory performances on the criteria belonging to the subset $T$ and completely unsatisfactory performances on the remaining criteria, $G \backslash T$. If the overall weight, $\mu(T)$, is different from the sum of weights, $\mu\left(\left\{g_{i}\right\}\right)$, of the criteria belonging to the subset $T$, this has to be interpreted as the result of some form of interaction among criteria. In practice only the interaction between a few number of pairs of criteria makes sense because the consideration of a large number of interacting criteria is difficult to be interpreted and leads to a huge cognitive effort from decision-makers. When taking into account a pair of criteria, $g_{i}$ and $g_{j}$, one of the following cases may occur:

- $\mu\left(\left\{g_{i}, g_{j}\right\}\right)=\mu\left(\left\{g_{i}\right\}\right)+\mu\left(\left\{g_{j}\right\}\right)$ : in this case there is no interaction between the two criteria, $g_{i}$ and $g_{j}$;

- $\mu\left(\left\{g_{i}, g_{j}\right\}\right)>\mu\left(\left\{g_{i}\right\}\right)+\mu\left(\left\{g_{j}\right\}\right)$ : in this case there is a mutualstrengthening effect, usually called synergy, between the two criteria, $g_{i}$ and $g_{j}$;

- $\mu\left(\left\{g_{i}, g_{j}\right\}\right)<\mu\left(\left\{g_{i}\right\}\right)+\mu\left(\left\{g_{j}\right\}\right):$ in this case there is a mutualweakening effect, usually called redundancy, between the two criteria, $g_{i}$ and $g_{j}$. 
A whole theory on interacting criteria phenomena and on how to measure such phenomena is now well established (mainly) following the work by Murofushi and Soneda (1993), which was generalized by Grabisch (1996b), axiomatized by Grabisch and Roubens (1999), and also studied by other authors, as for example, Fujimoto (2010), Fujimoto, Kojadinovic, and Marichal (2006), Grabisch, Marichal, and Roubens (2000), and Kojadinovic (2007b)

To apply the Choquet integral it is necessary to address two fundamental steps that can be described as follows: a) to assign utility values to the criteria performances on a common interval scale, and $b$ ) to build the capacity assigning a numerical value, $\mu(T)$, to each subset of criteria $T$, on a ratio scale. Many procedures have been proposed for both the aforementioned steps. In Grabisch and Labreuche (2016) and Grabisch (2016), the authors make use of MAсBETH method ( Bana e Costa, De Corte, \& Vansnick, 2012; 2016) to build both utility values and capacities. In the context of assigning capacities to subsets of criteria, another quite well-known approach is the regression method by Marichal and Roubens $\left(\begin{array}{lll}20 & 0 & 0\end{array}\right)$. This method seeks to build capacities compatible with some preference information provided by the decision-maker, such as a ranking of actions. This approach has been extended by Angilella, Corrente, and Greco (2015), Angilella, Greco, and Matarazzo (2010), in order to take into account the multi- plicity of capacities, which are compatible with the preference in- formation provided by the decisionmaker. Other approaches to identify the capacities are the least square method by Mori and Murofushi (1989), the minimum variance method by Kojadinovic (2007a), and a generalization of the least squares method by Meyer and Roubens (2005). With respect to the assignment of utility val- ues to criteria performances, one approach based on random sam- pling of values compatible with the preference information pro- vided by the decision-maker has been proposed by Angilella et al. (2015), Angilella, Greco, Lamantia, and Matarazzo (2004).

In the current paper, we propose a new technique for constructing the utilities (on an interval scale) and capacities (on a ratio scale) of the Choquet integral which is founded on the deck of cards method by Figueira and Roy (2002). The construction of the utilities of the performances of each criterion is based on the levels of the scale of the corresponding criterion, while the construction of the capacities is based on the definition of some adequate dummy projects. In the deck of cards method, each scale level/dummy project is written on a card, with some additional information if necessary. After, the decision-maker is asked to order the cards of scale levels/dummy projects, from the least important to the most important one. Then, for modeling the more or less "closeness" between two consecutive scale levels/dummy projects the decision-maker is asked to insert blank cards in the intervals that make a separation between such consecutive levels/dummy projects (for a complete description about the procedures see Section 3).

Blank cards in between successive scale levels/dummy projects are used to model the intensity of preferences, which are needed to construct interval and ratio scales. For such a purpose, we obviously could have chosen other methods, as for example, MAcBETH (Bana e Costa et al., 2012; 2016), but the choice of a deck of cards method was more or less imposed by the experts given their positive experience with this method in the previous study (see Bottero et al., 2015). They really appreciated the way of handling and manipulating the cards. In addition the concepts were very easy to introduce and understand. Of course, our method shares some similarities with MACBETH since both methods can be used to build interval scales for the utilities and ratio scales for the capacities. As for the latter, both methods make use of reference (dummy) projects. A brief comparison of MAcBETH and our deck of cards method will be presented in the conclusions.
It should be noticed that the purpose of this article is not to make a comparison of the results previously obtained in Bottero et al. (2015) with those we obtained with the current methodology. Our purpose is rather to test the potential of the Choquet integral in a real-world decision problem. This led us to propose two new procedures, one which allows to assign utility values on a common scale to the corresponding criteria performances, and the other which allows to assign numerical values to the capacities of the Choquet integral. From this perspective, we highlight the strengths and weaknesses of a decision aiding approach, which is based on the Choquet integral, where we first asked the experts to work separately, and then invited them to work together within a focus group context.

The work conducted with the experts allows to highlight the following aspects:

- The fact that it is difficult to establish robust conclusions with the Choquet integral when we want to take into account the sources of imperfect knowledge and arbitrariness inherent to the use of this approach.

- The fact the experts have been able, with no difficulties, to handle and be familiar with the deck of cards method for building interval scales as well as for making a comparison of projects and for building a ratio scale. In addition, this method proved to be very well adapted to facilitate the dialogue, collaboration, and interaction in the joint work performed by all the experts together. During this process, the method allowed to highlight the disagreement about the interpretations with respect to the meaning of some criteria.

These are indeed the two major conclusions of our work.

The paper is organized as follows. Section 2 presents a short description of the case study. Section 3 is devoted to the new procedures for building an interval scale with the objective of placing the performances of the criteria on a common utility scale, and to construct a ratio scale for assigning numerical values to the capac- ities of the Choquet integral. Section 4 is devoted to the way the two procedures were implemented in practice, i.e., the interaction with the experts and the focus group to assign numerical values to the required data for the application of the Choquet integral. Section 5 presents the design of the experiments, the construction of two specific sets of threshold functions, and the obtained results. Finally, Section 6 presents some conclusions according to the results obtained in the previous section and provides some direc tions for future research.

\section{The case study}

This section proposes a brief description of the context of the application. It then introduces the stakeholders and their representatives who participated in the process, illustrates the actions and criteria under analysis, and finally, discusses some aspects referring to the interaction between some pairs of criteria.

\subsection{The context}

The decision aiding problem under analysis concerns the evaluation of alternative projects for the requalification of an abandoned quarry located in Northern Italy. The quarry has been abandoned since 1975 and covers a total surface of $65,000 \mathrm{~m}^{2}$, with a depth of approximately $25 \mathrm{~m}$ from the ground level. From the environmental point of view, the area under analysis represents both a territorial weakness, due to its abandoned state that has caused uncontrolled vegetation growth and water-filled pits, and an opportunity, due to it being part of the Provincial ecological system of environmentally valuable sites. Five alternative projects (hereafter 
called actions) for the re-qualification of the area have been identi- fied and discussed by the Municipal Authority (for more details see Bottero, Ferretti, \& Pomarico, 2014).

In particular, the five re-qualification actions have been analyzed and compared in order to rank them from the best to the worst. The data concerning the actions as well as the performance criteria and their interaction effects come from two previous investigations carried out by the authors involved in the present research. The first study by Bottero et al. (2014) proposed the use of the Choquet integral to compare the alternative reuse actions for the abandoned quarry taking into account synergies (mutual-strengthening) and redundancies (mutual-weakening) among criteria. The second study, recently carried out by Bottero et al. (2015), adopted an extension of the ELECTRE III method proposed by Figueira et al. (2009) to model multiple interaction effects between criteria and tested it using the abandoned quarry re-qualification problem.

\subsection{The stakeholders and their representatives}

In public policy making the actors and their behaviors represent the core of any possible theoretical model (Boerboom \& Ferretti, 2014; Dente, 2014 ). The actors are those individuals or organizations that make the actions able to influence the decisional outcomes and do so because they pursue goals regarding the problem and its possible solution, or regarding their relations with other actors (Dente, 2014). In particular, any actor having a vested interest in the decision process, either directly affecting or being affected by its resolution, including experts and the public, is named a stakeholder. The first, essential, step of a decision aiding process to support public policy formulation thus consists of identifying the stakeholders and their objectives (e.g., Ferretti, 2016). In the present study, the decision to re-use the abandoned quarry is characterized by the presence of multiple stakeholders with different and frequently conflicting objectives. In what follows, the relevant stakeholders who can have a role in the process under investigation are presented for different administrative levels (for more details see Bottero et al., 2015).

1. National level: The Forestry Corps, i.e., the National Police Force in charge of the protection of natural heritage and landscape.

2. Regional level: (i) the Regional authority, i.e., the organisation responsible for territorial planning and management across the whole Piedmont Region, and (ii) the Regional Environmental Authority, i.e., the authority responsible for environmental protection in the area.

3. Provincial level: The Provincial authority, i.e., the authority responsible for territorial planning and management in the Province of Novara.

4. Local level: $(i)$ the municipal technical office, i.e., the authority responsible for the monitoring of all construction activities in the municipality, (ii) the mayor, i.e., the actor responsible for approving or rejecting any transformation project in the municipality, (iii) local practitioners, i.e., professionals such as architects and urban planners working in the area under analysis, (iv) inhabitants, i.e., the local population affected by the transformation, and finally, $(v)$ private entrepreneurs, i.e., private actors who could invest in the transformation projects.

The decision aiding process in this study was based on a participative approach that has been developed through the focus group technique (e.g., Morgan, 1988; Stewart \& Shamdasani, 1990). In order to include the concerns and points of view of the relevant stakeholders, three experts were involved in our focus group: an expert in the field of economic evaluation (expert e1); an expert in the field of environmental engineering (expert e2); an expert in the field of landscape ecology (expert e3). The use of a panel of experts expands the knowledge basis and may serve to avoid the possible biases that characterize the situation with a single expert. In our case, particular attention was thus dedicated to the panel composition in order to have it balanced and representative of the key stakeholders involved in the planning and evaluation process. It is worth highlighting that the experts with whom the authors of the paper worked in the present application are the same as in the study by Bottero et al. (2015).

\subsection{Actions, criteria, and the performance table}

Five alternative actions were considered by the Municipal Administration for the re-qualification of the abandoned quarry. All five actions consider the arrangement of security measures for the banks of the quarry but they differ regarding the specific recovery hypothesis.

In particular, the first action consists of basic reclamation of the quarry. In this project the quarry would be completely filled in with materials coming from building demolition and the topsoil would be left to the natural evolution of the vegetation.

The second action concerns the establishment of a new forest. More specifically, the project considers the complete filling in of the quarry with material coming from building demolition, the laying of topsoil and the establishment of a new oak-hornbeam wood.

The third action refers to the creation of a wetland in the area. Under this hypothesis the quarry would be partially filled in with material from building demolition work and the formation of a lake would be allowed, including the planting of wetland vegetation in the surrounding area.

The fourth action considers converting the quarry into a node of the provincial ecological network. This project consists of partially filling in the quarry with material from building demolition work, the formation of a lake and setting out pathways and recreational areas for visitors to the site.

The fifth action consists of developing a multi-functional structure. In this case, the quarry would be filled in to allow the construction of a recreational structure well integrated with the surrounding landscape and with high energy and environmental performances.

To summarise, the five actions under consideration are:
1. Basic reclamation $\left(a_{1}\right)$.
2. Valuable forest $\left(a_{2}\right)$.
3. Wetland $\left(a_{3}\right)$.
4. Ecological network $\left(a_{4}\right)$.
5. Multi-functional area $\left(a_{5}\right)$.

To support comparison of the five actions for the requalification of the quarry, a family of six criteria has been built. The criteria represent all the concerns pertaining to the sustainability of the decision aiding problem and they can be summarized as follows:

1. Investment costs $\left(g_{1}\right)$ : This criterion considers the building costs for carrying out the re-qualification project; the scale unit is Euros and this criterion is to be minimized.

2. Profitability $\left(g_{2}\right)$ : This criterion evaluates the income that the investment is likely to generate on the local economic system; the criterion scale is a seven-level qualitative scale where level 1 means "very bad", 2 "bad", 3 "rather bad", 4 "average, 5 "rather good", 6 "good", and 7 "very good"; this criterion is to be maximized.

3. New services for the population $\left(g_{3}\right)$ : This criterion concerns the effects of the re-qualification projects in terms of generating new services for the local population, including sport facilities, recreational areas, green parks, etc; its scale is the aforementioned seven-level qualitative scale and it is to be maximized. 
Table 1

\begin{tabular}{|c|c|c|c|c|c|c|}
\hline & $\begin{array}{l}\text { Costs } \\
\left(g_{1}\right)\end{array}$ & $\begin{array}{l}\text { Profitability } \\
\left(g_{2}\right)\end{array}$ & $\begin{array}{l}\text { Services } \\
\left(g_{3}\right)\end{array}$ & $\begin{array}{l}\text { Surface } \\
\left(g_{4}\right)\end{array}$ & $\begin{array}{l}\text { Environment } \\
\left(g_{5}\right)\end{array}$ & $\begin{array}{l}\text { Consistency } \\
\left(g_{6}\right)\end{array}$ \\
\hline$a_{1}$ & 30,000 & Rather bad (3) & Very bad (1) & 2 & Average (4) & Yes (1) \\
\hline$a_{2}$ & 45,000 & Rather bad (3) & Rather good (5) & 5 & Rather good (5) & Yes (1) \\
\hline$a_{3}$ & 90,000 & Very bad (1) & Good (6) & 3.2 & Very good (7) & Yes (1) \\
\hline$a_{4}$ & 120,000 & Very bad (1) & Very good (7) & 3.5 & Good (6) & Yes (1) \\
\hline$a_{5}$ & 900,000 & Very good (7) & Very good (7) & 1 & Rather bad (3) & No $(0)$ \\
\hline
\end{tabular}

4. Naturalized surface $\left(g_{4}\right)$ : This criterion considers the impacts of the projects on the landscape quality and the conservation of bio-diversity; its scale is in hectares of naturalized surface and it is to be maximized.

5. Environmental effects $\left(g_{5}\right)$ : This criterion considers the consequences that the projects have on the environmental system, including minimization of geo-technical and hydro-geological impacts; its scale is the aforementioned seven-level qualitative scale and it has to be maximized.

6. Consistency with local planning requirements $\left(g_{6}\right)$ : This criterion considers the existence of urban planning constraints that could affect the administrative feasibility of the project; the criterion scale distinguishes between two situations, i.e., feasible or "yes" (corresponding to level 1) and not feasible or "no" (corresponding to level 0); this criterion is to be maximized.

The performances of the actions under analysis according to the considered criteria are presented in Table 1.

\subsection{Why do some criteria interact?}

Two different forms of interaction between criteria have been considered in the present application: the mutualstrengthening effect and the mutual-weakening effect (Figueira et al., 2009). A mutual-strengthening effect between two criteria is present when the overall weight of these two criteria is greater than the sum of the weights of the two criteria considered separately, while a mutual-weakening effect between two criteria is present when their overall weight is less than the sum of the weights of the two criteria considered separately.

In the context of the present application, starting from the interaction between criteria that were identified in the previous study (Bottero et al., 2015), the research considers the following interactions:

\footnotetext{
- Mutual-strengthening between criteria $g_{1}$ (costs) and $g_{5}$ (environment).

- Mutual-weakening between criteria $g_{4}$ (surface) and $g_{5}$ (environment).
}

In particular, according to the experts involved in the focus group to discuss the interaction between the evaluation criteria, there is a mutual-strengthening effect between criteria $g_{1}$ (costs) and $g_{5}$ (environment): indeed, a project with negative environmental effects very often also has low costs (due for example to the use of cheap technology with pollutant emissions). Therefore, a project with positive environmental effects and low costs is very well appreciated. Consequently, the overall weight of this pair of criteria is greater than the sum of their individual weights.

Moreover, during the focus group the experts identified a mutual-weakening effect between the criteria $g_{4}$ (surface) and $g_{5}$ (environment): indeed, a project of high ecological quality is often also of high environmental quality. Consequently, the overall weight of this pair of criteria is smaller than the sum of their individual weights.
It should be highlighted that the antagonistic effect identified in the focus group for the ELECTRE III application has not been considered in the present research as this effect cannot be modeled using the basic Choquet integral approach. An antagonistic effect occurs when a criterion favoring an action is opposing to a criterion favoring another action; the interaction between the two criteria leads to a reduction of the weight of the criterion favoring the first action by an opposing power of the criterion favoring the second one. It is, however, possible to model such an effect by applying the bipolar Choquet integral (Grabisch \& Labreuche, 2005; Greco \& Rindone, 2014). To simplify the interaction protocol with the experts, we used the basic Choquet integral.

\section{Choquet integral and procedures for determining capacities and common scales}

This section presents fundamental concepts about the Choquet integral. This aggregation function requires the assignment of a weight to each subset of criteria by means of a function called capacity. Moreover, the Choquet integral also requires that the evaluations or utilities of each action on the considered criteria are expressed on the same scale. New procedures for computing capacities and utilities on a common scale are also introduced in this section.

\subsection{The Choquet integral}

Let $A$ denote a set containing $m$ actions, $a_{1}, \ldots, a_{m}$, and $G$ denote a set with $n$ criteria, $g_{1}, \ldots, g_{i}, \ldots, g_{n}$ (for the sake of simplicity we can also identify the criteria by their indices). For an action $a$ and a criterion $g_{i}, g_{i}(a)$ is the performance of action $a$ on criterion $g_{i}$, and $u_{i}\left(g_{i}(a)\right)$ is the utility of the performance $g_{i}(a)$; again for the sake of simplicity we will henceforth use $u_{i}(a)$ instead of ui $\left(g_{i}(a)\right)$.

The Choquet integral (Choquet, 1953) is an aggregation function that permits the aggregation of utilities on the considered criteria taking into account interactions among criteria. It is based on the concept of capacity or fuzzy measure (see Grabisch, 1996a). A ca pacity is a set function, $\mu: 2^{G} \rightarrow[0,1]$, on the power set, $2^{G}$ (i.e., the set of all subsets of $G$ ) satisfying the following properties:

i) $\mu(\emptyset)=0$ and $\mu(G)=1$ (boundary conditions).

ii) $\forall S \subseteq T \subseteq G \mu(S) \leq \mu(T)$ (monotonicity condition).

For any subset $T \subseteq G$, the value $\mu(T)$ represents the capacity (weight) of the criteria belonging to the subset $T$. This can be interpreted as the utility value of an action with totally satisfactory performances (i.e., that correspond to a utility value of 1 ) on the criteria belonging to the subset $T$, and with totally unsatisfactory performances (i.e., that correspond to a utility value of 0 ) on the remaining criteria.

Since in any case $\mu(\emptyset)=0$ and $\mu(G)=1$, the values $\mu(S)$ assigned by the capacity $\mu$ to all other $2^{|G|}-2$ subsets $S$ of $G$ have to be defined. For the sake of simplicity, we call the values $\mu(S)$ as 
the capacities of set $S$. Given an action $a \in A$ and a capacity $\mu$ on $2^{G}$, the Choquet integral can be defined as follows:

$C_{\mu}(a)=\sum_{i=1}^{n}\left(u_{(i)}(a)-u_{(i-1)}(a)\right) \mu\left(G_{i}\right)$,

where $u_{(1)}, \ldots, u_{(n)}$ are the utilities of criteria from $G$, reordered in such a way that $u_{(1)}(a) \leq \cdots \leq u_{(i)}(a) \leq \cdots \leq u_{(n)}(a)$, and $G_{i}=$ $\{(i), \ldots,(n)\}$, for $i=1, \ldots, n$, with $u_{(0)}(a)=0$.

Given a capacity $\mu$ on $2^{G}$, its Möbius representation is a function $m: 2^{G} \rightarrow \mathbb{R}$ such that, for all $S \subseteq G$,

$\mu(S)=\sum_{T \subseteq S} m(T)$,

we have that,

$m(S)=\sum_{T \subseteq S}(-1)^{|S-T|} \mu(T)$,

and the above properties ( $i$ ) and (ii) can be reformulated as follows,

$$
\begin{aligned}
& \left.i^{\prime}\right) m(\emptyset)=0, \quad \sum_{T \subseteq G} m(T)=1 . \\
& \left.i i^{\prime}\right) \forall i \in G \text { and } \forall R \subseteq G \backslash\{i\}, \quad m(\{i\})+\sum_{T \subseteq R} m(T \cup\{i\}) \geqslant 0 .
\end{aligned}
$$

The Choquet integral can now be expressed in terms of the Möbius representation $m$ of the capacity $\mu$ as follows,

$C_{\mu}(a)=\sum_{T \subseteq G} m(T) \min _{i \in T}\left\{u_{i}(a)\right\}$.

\subsection{Interaction between criteria}

The key reason to use the Choquet integral is the possibility to take into account interaction between criteria. If there is no interaction between the criteria belonging to the subset $R$ and the criteria belonging to the subset $S$ (with $R \cap S=\emptyset$ ) the utility value, $\mu(R \cup S)$, of an action with a utility value 1 on the criteria belonging to the subset $R \cup S$ and a utility value 0 on the other criteria, should be equal to the sum of $\mu(R)$ (the utility value of an action with a utility value 1 on the criteria belonging to the subset $R$ and a utility value 0 on the remaining criteria) plus $\mu(S)$ (the utility value of an action having a utility value 1 on the criteria belonging to the subset $S$ and a utility value 0 on the remaining criteria), i.e., $\mu(R \cup S)=\mu(R)+\mu(S)$.

Taking into account pairs of criteria $g_{i}, g_{j} \in G$, one can distinguish between

- mutual-strengthening effect (synergy) between $g_{i}$ and $g_{j}$ in case $\mu(\{i, j\})>\mu(\{i\})+\mu(\{j\})$, that is, in terms of Möbius representation, $m(\{i, j\})>0$;

- mutual-weakening effect (redundancy) between $g_{i}$ and $g_{j}$ in case $\mu(\{i, j\})<\mu(\{i\})+\mu(\{j\})$, that is, in terms of Möbius representation, $m(\{i, j\})<0$;

- absence of interaction between $g_{i}$ and $g_{j}$ in case $\mu(\{i, j\})=$ $\mu(\{i\})+\mu(\{j\})$, that is, in terms of Möbius representation, $m(\{i, j\})=0$.

Therefore, in case of interaction only between pairs of criteria $g_{i}, g_{j} \in G, m(\{i, j\})$ contains all the related information, and in fact, in this case $m(\{i, j\})$ corresponds to the interaction index. It has to be noted that for the general case, when one can have interactions for any subsets of criteria, the situation is different. However, also for this case, there exists a definition of interaction between criteria that can be measured with a general formulation of the interaction index (Grabisch, 1997).

In the following we consider only interaction between pairs of criteria. Let $O$ denote the set of interacting pairs of criteria, $\{i, j\}$.
Moreover, for the sake of simplicity, we will use $m_{i}$ instead of $m(\{i\})$ for all $i \in G$ and $m_{i j}$ instead of $m(\{i, j\})$ for all $\{i, j\} \in O$. The same applies to $\mu_{i}$ and $\mu_{i j}$.

In real-world decision problems, it seems reasonable to focus on the interactions of a small set of pairs of criteria. Thus, for all $S$ $\subseteq G$ we have

$\mu(S)=\sum_{i \in S} m_{i}+\sum_{\{i, j\} \subseteq S,\{i, j\} \in O} m_{i, j}$,

and,

$\mu(G)=\sum_{i \in G} m_{i}+\sum_{\{i, j\} \in O} m_{i, j}=1$,

so that the Choquet integral can be reformulated as

$C_{\mu}(a)=\sum_{i \in G} m_{i} u_{i}(a)+\sum_{\{i, j\} \in O} m_{i, j} \min \left\{u_{i}(a), u_{j}(a)\right\}$.

It is possible to observe that Eq. (5) corresponds to a specific case of the 2-additive capacity proposed by Grabisch (1997). Indeed, a 2-additive capacity considers interactions only for pairs of criteria. In Eq. (5) the interactions are limited to the specific pairs of criteria in the subset 0 , while there is no interaction for the remaining pairs of criteria.

Example 1. Let us consider only two interacting pairs of criteria, $\{i$, $j\}$ (characterized by a mutual-strengthening effect) and $\{k, j\}$ (characterized by a mutual-weakening effect). From the sign of these interaction effects and property $\left.i i^{\prime}\right)$, we have
a) $m_{i j} \geqslant 0$
c) $m_{i}+m_{i j} \geqslant 0$
e) $m_{k}+m_{k j} \geqslant 0$
g) $m_{j}+m_{i j}+m_{k j} \geqslant 0$
$\begin{array}{ll}\text { b) } & m_{k j} \leqslant 0 \\ \text { d) } & m_{j}+m_{i j} \geqslant 0 \\ \text { f) } & m_{j}+m_{k j} \geqslant 0\end{array}$

From the Möbius representation (3) we have $m_{i}=\mu_{i}, m_{j}=\mu_{j}$, $m_{k}=\mu_{k}, m_{i j}=\mu_{i j}-\mu_{i}-\mu_{j}$, and $m_{k j}=\mu_{k j}-\mu_{k}-\mu_{j}$. By replacing them in the previous system we obtain the following two consistency conditions,

$\mu_{i j} \geqslant \mu_{i}+\mu_{j}$

and

$\max \left\{\mu_{k}, \mu_{j}\right\} \leqslant \mu_{k j} \leqslant \mu_{k}+\mu_{j}$.

The first condition comes from (a) while the second comes from (b), (e), (f), and (g) (note that from (g), $\mu_{k j} \geqslant \mu_{i}+\mu_{k}+$ $\mu_{j}-\mu_{i j}$, but since from $(a), \mu_{i}+\mu_{j}-\mu_{i j} \leqslant 0$, a non-negative amount is removed from $\mu_{k}$; we can thus discard the expression obtained from $(g)$ ).

\subsection{A common scale for criteria utilities}

A fundamental requirement when applying the Choquet integral is that the criteria utilities must be on the same scale. Indeed, in formulations (1), (4), and (7), the computation of the Choquet integral requires a comparison of the utilities on all the criteria. More precisely, when applying formulation (1) it is necessary to rank order the utilities of criteria in $G$, from the smallest to the largest, and to compute the differences of utilities on the different criteria, while when applying formulations (4) and (7) it , is necessary to compute the minimum of the utilities for all pairs of interacting criteria. Therefore, a fundamental condition for the application of the Choquet integral is that the utilities on the considered criteria have to be expressed on a common scale.

\subsection{Determining the capacities}

Figueira and Roy (2002) proposed a modified version of Simos' deck of cards method for determining the weights of criteria 
within the context of outranking methods (for a list of applications see Siskos \& Tsotsolas, 2015). In the conclusion of their work Figueira and Roy (2002) stated that the method could be adapted to build other ratio scales as well as to build interval scales. The purpose of the current and the next subsection is to present two extensions of the deck of cards method for building ratio and interval scales, respectively. With such a purpose in mind, instead of considering criteria, we can take into account more general objects (for instance, actions, projects, scale levels). In any case, the dia$\log$ between the analyst(s) and the decision-maker(s) or expert(s) should take into account the following aspects:

1. The analyst should provide the decision-maker with a first set of cards, each one containing the name of each object and some additional information (if necessary).

2. The analyst should also provide the decision-maker with a large enough set of blank cards.

3. The analyst must then ask the decision-maker for a ranking of the cards in the first set - the decision-maker should rank the objects from the least to the most important (if some are tied, they should occupy the same position in the ranking).

4. The analyst should call the attention of the decision-maker to the fact that two consecutive positions in the ranking may be more or less close. This greater or smaller closeness can be modeled through the insertion of blank cards in the intervals of the consecutive positions in the ranking.

5. Finally, the analyst should obtain from the decision-maker information allowing to fix the ratio $z$ between the value of the most appreciated and the value of the least appreciated object (i.e., how many times the most appreciated object is more important than the least appreciated one). Two other reference objects can be considered for the definition of this ratio.

It should be highlighted that the information obtained in the last point is important in order to build ratio scales. When building interval scales, such an information about the ratio $z$ is replaced by the definition of at least two reference values, as can be seen in Section 3.5.

The construction of a ratio scale for capacities requires, in general, the consideration of very specific objects, which we will call projects. Marichal and Roubens (2000) proposed a method for determining the capacities of the Choquet integral from a reference set (of projects); this method can be viewed as an extension of the swing weighting procedure (see page 275 in von Winterfeldt \& Edwards, 1986 ). Based on their idea, and assuming that the minimal and the maximal utilities on each criterion are 0 and 1, respectively, we propose to build the reference set of projects as follows:

- $n$ projects (as many as the number of criteria), denoted by $p_{j}$, for all $j \in G$, which can be characterized by a vector of the form $\left(0, \ldots, 0, u_{j}\left(p_{j}\right)=1,0, \ldots, 0\right)$, where $p_{j}$ has the highest evaluation on criterion $j$ and the lowest elsewhere.

- $|O|$ projects (as many as the number of interacting criteria pairs) denoted by $p_{k}=p_{i j}, k=n+1, \ldots, n+|O|$, which can also be characterized by a vector of the form $\left(0, \ldots, 0, u_{i}\left(p_{i}\right)=\right.$ $\left.1,0, \ldots, 0, u_{j}\left(p_{j}\right)=1,0, \ldots, 0\right)$, for all $\{i, j\} \in O$, where $p_{k}$ has the highest utilities on criteria $i$ and $j$, and the lowest elsewhere.

The method we propose for assessing the capacity, $\mu$, necessary to compute the Choquet integral can be presented, as a step-bystep procedure, as follows (it also allows computing the Möbius representation $m$ of the capacity $\mu$ ):

1. Consider the following finite set of reference projects: $P=$ $\left\{p_{1}, p_{2}, \ldots, p_{k}, \ldots, p_{t}\right\}$ (where $t=n+|O|$ ).

2. Consider the ranking of the projects provided by the decisionmaker and denoted by $R_{1}, \ldots, R_{h}, \ldots, R_{v}$, the equivalence classes in the ranking $\left(R_{1}\right.$ containing the least preferred projects, $R_{2}$ containing the second least preferred projects, and so on, until $R_{v}$, containing the most preferred projects). Let us denote by $r_{h}$ a project representative of projects in the equivalence class $R_{h}, h=1, \ldots, v$. Of course, all the projects in class $R_{h}$ will have the same value of $r_{h}$. Let $e_{h}$ denote the number of blank cards between the equivalence classes $R_{h}$ and $R_{h+1}$, $h=1, \ldots, v-1$. Note that in the ranking there are as many units between the first and the last position as the total number of blank cards plus the number of intervals in the ranking.

3. Assign a value to project $r_{1}$ (and consequently to all the projects in $R_{1}$ ), say $w\left(r_{1}\right)=\ell$ (it is frequent to consider $w\left(r_{1}\right)=$ 1 ) (we assume that none of the projects has utility 0 ).

4. Compute the value of each unit as follows:

$\alpha=\frac{\ell(z-1)}{s}$

where

$s=\sum_{h=1}^{v-1}\left(e_{h}+1\right)$,

that is, $\alpha$ is obtained by dividing the difference between the values of the most preferred objects $\left(w\left(r_{v}\right)=\ell z\right)$ and the least preferred objects $\left(w\left(r_{1}\right)=\ell\right), s$ being the number of units between $R_{1}$ and $R_{v}$ (if two other reference projects are taken into account these formulas must be modified accordingly).

5. Compute the values $w\left(r_{h}\right), h=2, \ldots, v$, as follows:

$$
w\left(r_{h}\right)=\ell+\alpha\left(\sum_{j=1}^{h-1}\left(e_{h}+1\right)\right)
$$

6. Compute the value of each project, $w\left(p_{k}\right)=w\left(r_{h}\right)$ for all $p_{k} \in R_{h}, h=1, \ldots, v$.

7. Compute the modified values $\bar{w}\left(p_{k}\right)$ as follows: $\bar{w}\left(p_{k}\right)=w\left(p_{k}\right)$ if $k=i \in G ; \bar{w}\left(p_{k}\right)=w\left(p_{k}\right)-w\left(p_{i}\right)-w\left(p_{j}\right)$ if $p_{k}=p_{i j},\{i, j\} \in$ 0 , for $k \geqslant n+1$.

8. Compute the Möbius coefficients, $m_{k}$, and the capacities, $\mu_{k}$, for $k=1, \ldots, t$ :

$$
m_{k}=\frac{\bar{w}\left(p_{k}\right)}{\sum_{j=1}^{t} \bar{w}\left(p_{j}\right)}
$$

and

$$
\mu_{k}=\frac{w\left(p_{k}\right)}{\sum_{j=1}^{t} \bar{w}\left(p_{j}\right)},
$$

where the coefficients $m_{k}$ must fulfill conditions $i^{\prime}$ ) and $i i^{\prime}$ ) and must be consistent with the sign of the interactions (mutual-strengthening and mutual-weakening) provided by the decision-maker. Otherwise, a non-conformity case occurs.

In Section 4, we will show how the method proposed in this section can be applied to elicit the interaction aspects with the experts in order to define the values for the capacities of the Choquet integral.

\subsection{Building interval scales}

The utility values of the Choquet integral are the levels of a common interval scale, in general, within the range $[0,1]$. The translation from the original scales of the criteria to a single common interval scale requires the use of a procedure that should account for the intensity of preferences between consecutive intervals of the scale. In this section, we present a new procedure 
for defining an interval scale based on the concepts of the deck of cards method (Figueira \& Roy, 2002). The procedure presented here allows scales not necessarily within the range $[0,1]$ to be constructed.

In order to build an interval scale we need to define at least two reference levels (instead of the definition of $z$, as in the case of ratio scales), to anchor the computations. If more than two reference levels are defined, we can replicate the procedure for every two consecutive reference levels.

1. Consider the following discrete scale of criterion $g$ : $E_{g}=$ $\left\{l_{1}, l_{2}, \ldots, l_{k}, \ldots, l_{t}\right\}$, where $l_{1} \prec l_{2} \prec \cdots \prec l_{k} \prec \cdots \prec l_{t-1} \prec l_{t} \quad(\prec$ means "strictly less preferred than").

2. Define two reference levels, say $l_{p}$ and $l_{q}$ and assign two utility values to these reference levels. We frequently use

$u\left(l_{p}\right)=0$.

$u\left(l_{q}\right)=1$.

Other values can be assigned to $l_{p}$ and $l_{q}$. Observe that very often levels $l_{p}$ and $l_{q}$ coincide with $l_{1}$ and $l_{t}$, respectively.

3. Consider the ranking of the levels with a certain number of blank cards, $e_{k}$, in the intervals between every two consecutive levels, $l_{k}$ and $l_{k+1}, k=1, \ldots, t-1$ :

$l_{1} e_{1} l_{2} e_{2} \ldots l_{p} e_{p} l_{p+1} e_{p+1} \ldots l_{k} e_{k} l_{k+1} \ldots l_{q-1} e_{q-1} l_{q} \ldots l_{t-1} e_{t-1} l_{t}$.

4. Now, consider only the levels in between $l_{p}$ and $l_{q}$ (in between levels $l_{k}$ and $l_{k+1}$ there are $\left(e_{k}+1\right)$ units) and compute the unit valuation:

$\alpha=\frac{u\left(l_{q}\right)-u\left(l_{p}\right)}{h}$,

where

$h=\sum_{k=p}^{q-1}\left(e_{k}+1\right)$,

which represents the number of units between levels $l_{p}$ and $l_{q}$.

5. Compute the utility value, $u\left(l_{k}\right)$, for each level, $k=1, \ldots, t$, as follows:

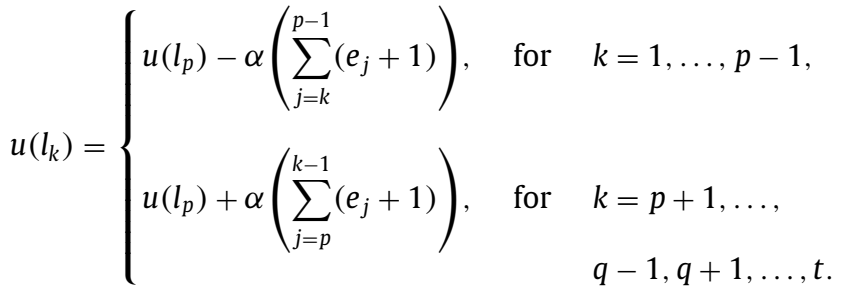

In Section 4, we will present the details about the interaction elicitation protocol used with the experts to define the common scale, starting from the original quantitative and verbal scales. The original scales were encoded in a common $[0,1]$ utility scale, but for some scales we defined three reference levels with the utility values of $0,0.5$, and 1 . The previous procedure has been applied twice, to encode utility values within the range $[0,0.5]$, and then within the range [0.5,1]. The formula of Point 5 above becomes simply,

$u\left(l_{k}\right)=u\left(l_{1}\right)+\alpha\left(\sum_{j=1}^{k-1}\left(e_{j}+1\right)\right)$, for $k=2, \ldots, t$,

with $u\left(l_{1}\right)=0$ in the first subinterval and $u\left(l_{1}\right)=0.5$ in the second.

An attempt to build an interval scale was also proposed by Pictet and Bollinger (2008), but without considering the value of the unit and considering instead the blank cards as positions in the ranking. This makes the method unpractical when confronted with a large number of blank cards in the intervals between consecutive levels. In addition, the formula to compute the values of each level cannot assign values strictly lower than the value of the lower reference level.

\section{Assigning numerical values to the required data for the application of the Choquet integral}

For the assignment of a utility value to each one of the considered actions through the application of the Choquet integral it is necessary:

1. To place on a common utility scale the performances of the actions according to each one of the considered criteria. We will describe in Section 4.1 the procedure we followed when the performances were characterized on a verbal scale. In Section 4.2 we will deal with the criteria when the performances are characterized on numerical (continuous) scales. As noted above, this common utility scale must be an interval scale and the utility values thus considered should be commensurable, i.e., these values should be such that for whatever the action $a$ and the criteria $g_{i}$ and $g_{j}$ considered, every equality of the type,

$u_{i}(a)=u_{j}(a)$,

is supposed to have the following meaning: The intensity of satisfaction provided by the action $a$ on criterion $g_{i}$ is the same as that provided by this action on criterion $g_{j}$.

2. To assign a numerical value to each capacity of the Choquet integral, which is the object of Section 4.3.

The assignment of these numerical values in a perfectly rigourous manner would require posing a very large number of questions to the experts. The time they have and especially the risk of tiredness restricts the number of questions which it is possible to ask them. This led us to take into account a certain number of empirical hypotheses without being able to verify them.

\subsection{Assigning utility values to the verbal scale levels}

Concerning criterion $g_{6}$ (consistency) we have assigned a utility 1 to the verbal level "yes" and 0 to "no" (see Section 2.3).

Concerning the seven levels of the verbal scales associated with criteria $g_{2}$ (profitability), $g_{3}$ (services), and $g_{5}$ (environment), we have assigned:

- A utility value 1 to the scale level "very good".

- A utility value 0.5 to the scale level "average".

- A utility value 0 to the scale level "very bad".

When working with the experts we started by explaining that utility value $u_{j}(a)$ of action $a$, according to criterion $g_{j}$, should be interpreted as the intensity of satisfaction provided by action $a$ with respect to this criterion. Utility value 1 is associated with a performance that provides a total intensity of satisfaction: no better performance level would be likely to improve this intensity of satisfaction. Utility value 0 is associated with a performance level that provides a total intensity of dissatisfaction: all the performances levels below this level provide the same total intensity of dissatisfaction.

Subsequently, we called the attention of the experts to the meaning of Eq. (10). Finally, we let them work separately to assign a utility value to each one of the four intermediate scale levels. The experts have admitted (with some reservations from expert e2) that, for each of these intermediate levels, the utility values should be the same whatever the criterion considered, among the three that share this seven-level verbal scale. Abandoning this hypothesis would mean applying the procedure presented hereafter 
Table 2

Number of blank cards in the intervals between consecutive levels by expert.

\begin{tabular}{|c|c|c|c|c|c|c|c|c|c|c|c|c|c|}
\hline Experts & $l_{1}(\mathrm{vb})$ & $e_{1}$ & $l_{2}(\mathrm{~b})$ & $e_{2}$ & $l_{3}(\mathrm{rb})$ & $e_{3}$ & $l_{4}(\mathrm{a})$ & $e_{4}$ & $l_{5}(\mathrm{rg})$ & $e_{5}$ & $l_{6}(\mathrm{~g})$ & $e_{6}$ & $l_{1}(\mathrm{vg})$ \\
\hline e1 & & 0 & & 0 & & 0 & & 0 & & 0 & & 0 & \\
e2 & & 3 & & 2 & & 1 & & 1 & & 2 & & 3 & \\
e3 & & 5 & & 3 & & 7 & & 7 & & 3 & & 5 & \\
\hline
\end{tabular}

Table 3

Utility values for the scale levels by encoding (expert).

\begin{tabular}{lllllllll}
\hline Experts & Encoding & $u(v b)$ & $u(b)$ & $u(r b)$ & $u(a)$ & $u(r g)$ & $u(g)$ & $u(v g)$ \\
\hline e1 & b1 & 0.0000 & 0.1667 & 0.3333 & 0.5000 & 0.6667 & 0.8333 & 1.0000 \\
e2 & b2 & 0.0000 & 0.2222 & 0.3889 & 0.5000 & 0.6111 & 0.7778 & 1.0000 \\
e3 & b3 & 0.0000 & 0.1667 & 0.2778 & 0.5000 & 0.7222 & 0.8333 & 1.0000 \\
\hline
\end{tabular}

three times successively (the justification for this can be found in Section 3.5). Furthermore, abandoning this hypothesis would increase considerably the number of computations to be performed. As will be seen in Section 5.3.1, this hypothesis is with no consequences.

We gave the expert eh $(h=1,2,3) 7$ cards, each carrying the name of each one of the seven levels. These cards were ranked according to the order of the scale levels. The expert eh was invited to work on the lower part of the verbal scale, then on its upper part. We also provided the expert with 15 blank cards for each part of the scale. The expert was asked to insert blank cards in each of the intervals of the lower part of the scale in such a way that the number of cards introduced should represent the higher or lower intensity of satisfaction between two consecutive levels. The same procedure was applied to the upper part of the scale.

The experts reacted in the following way when applying this procedure:

- At first, they were unsure ( i.e., they hesitated) as to the differ- ences between two consecutive levels because in their minds all of these differences were equal.

- Then, when trying to insert the blank cards in the different intervals, they acknowledged (except for e1) that these differences could not be all equal.

- It should be noted that the three experts appreciated both parts of the scale in a symmetric way. Nevertheless, their utilities are contrasted (see Table 2: in this table $l_{1}$ means "very bad", $l_{2}$ means "bad", and so on. For the sake of readability the initials of the verbal levels are also in brackets).

This led us to introduce three possible encodings (by applying the method of Section 3.5), denoted by b1, b2, and b3, for the seven-level verbal scale. Each of them is linked to an expert e1, e2, and e3, respectively (see Table 3 ).

The utility value of each action with respect to criteria $g_{2}, g_{3}$, $g_{5}$, and $g_{6}$, for each one of the three encodings above is presented in Table 5 in Appendix A.

\subsection{Assigning utility values to the numerical (continuous) scale levels}

Two criteria are concerned: $g_{1}$ (the investment cost) and $g_{4}$ (the surface of the naturalized area). For these two criteria, the performances used to characterize the 0 and 1 utility values cannot be defined in an obvious way. For each one of the two criteria we considered two possible encodings.

a) With respect to the cost:
- Option 1: Utility value 1 is defined by the cost of the less expensive action (30,000 Euro), and utility value 0 is defined by the cost of the most expensive action (900,000 Euro).

- Option 2: Utility value 1 is defined by $70 \%$ of the cost of the less expensive action (21,000 Euro), and utility value 0 is defined by $130 \%$ of the cost of the most expensive action (1,117,000 Euro).

b) With respect to the surface:

- Option 1: Utility value 1 is defined by the action that leads to the naturalization of the largest surface ( $5 h a)$, and utility value 0 is defined by the action that leads to the naturalization of the lowest surface ( $1 \mathrm{ha}$ ).

- Option 2: Utility value 1 is defined by the largest surface that could be naturalized ( $6.5 \mathrm{ha})$, and utility value 0 is defined by the lowest surface that could be naturalized $(0 \mathrm{ha})$.

On this basis we defined three encodings for each criterion. The first two encodings, denoted by $\mathrm{c} 1$ and $\mathrm{c} 2$, for the cost criterion $\left(g_{1}\right)$, and by $\mathrm{s} 1$ and $\mathrm{s} 2$, for the surface criterion $\left(g_{4}\right)$, are defined by taking into account the above Options 1 and 2 . The utility values assigned to the intermediate performances of criterion $g_{j}(j=1,4)$ in a given subinterval $\left(g_{j}^{\ell}<g_{j}<g_{j}^{u}\right)$ are defined by linear interpolation, through the application of the following formula:

$u_{j}\left(g_{j}\right)=u_{j}\left(g_{j}^{\ell}\right)+\frac{g_{j}-g_{j}^{\ell}}{g_{j}^{u}-g_{j}^{\ell}}\left(u_{j}\left(g_{j}^{u}\right)-u_{j}\left(g_{j}^{\ell}\right)\right)$.

These encodings were presented to the experts, who considered them relevant despite the element of arbitrariness they may contain. Then, we proposed that the experts work collectively to define only two other new encodings (c3 and s3) to avoid multiplying the number of questions and to reduce fatigue for the experts. Only Option 2 was considered to define the performances characterizing extreme utility values of these new encodings (other options could be introduced later to take into account more encodings if it was proved to be necessary).

We started by asking the experts to work together to define encoding s3. The following procedure was used. We first reminded the experts that the surface of $6.5 \mathrm{ha}$ had been chosen to characterise a total intensity of satisfaction, and 0 ha to characterize a total intensity of dissatisfaction. Then, we asked them to define a surface $m$ that was able to characterize an average level of intensity of satisfaction in the considered scale. We added that an average level indicates a surface $m$ such that moving from 0 ha to $m$ ha brings the same variation of intensity of satisfaction as the variation that brings the transition from $m$ ha to $6.5 \mathrm{ha}$. We asked them if $m=3.25 \mathrm{ha}$ (the middle of the interval) would be suitable. They judged this value very high, and after some discussion they 
proposed $m=3 \mathrm{ha}$. We then continued the same exercise by taking the interval $0 \mathrm{ha}-3 \mathrm{ha}$; and, finally we did the same for the interval $3 h a-6$. $5 h a$. The experts quickly agreed to keep the values $1 \mathrm{ha}$ and $5 \mathrm{ha}$, respectively. In each of these intervals thus defined, we proceeded by linear interpolation to assign a utility value to each of the considered surfaces.

Concerning the definition of encoding c3 we asked the experts to work in the same way as in the previous case. However, they found this new exercise much more difficult given the large difference of the costs that characterize the utility values 1 and 0, 21,000 Euro and 1,117,000 Euro, respectively. Nevertheless, a general agreement was reached to keep the values 150,000 Euro, 400,000 Euro, and 800,000.

For each action the utility values associated with their performances on criteria $g_{1}$ and $g_{4}$ were defined by linear interpolation, for each of the three considered encodings (see Table 6 in Appendix A ).

\subsection{Assigning numerical values to the capacities of the Choquet integral}

We followed the procedure described in Section 3.4. A mutualstrengthening effect and a mutual-weakening effect being taken into account, eight capacities must be considered. This is the reason that led us to introduce eight types of dummy projects, as follows:

- Projects of type $p_{j}$, for $j=1, \ldots, 6$, which are characterized by a utility value of 1 on criterion $g_{j}$, and a utility value of 0 on all other criteria.

- Project of type $p_{7}$ (also denoted by $p_{45}$ ), which is characterized by a utility value of 1 on criteria $g_{4}$ and $g_{5}$, and a utility value of 0 on all other criteria.

- Project of type $p_{8}$ (also denoted by $p_{15}$ ), which is characterized by a utility value of 1 on criteria $g_{1}$ and $g_{5}$, and a utility value of 0 on all other criteria.

For the characterization of each type of project it is necessary to take into account the actual performances that are encoded by the 0 and 1 utility values with respect to the considered project. Concerning criteria $g_{2}, g_{3}, g_{5}$, and $g_{6}$, such performances are those corresponding to the extreme levels of the verbal scales. For each of the two remaining criteria, $g_{1}$ and $g_{4}$, two options were envisaged (see Section 4.2). They correspond to encodings $c 1, c 2$, and $s 1$, $\mathrm{s} 2$, respectively. This lead us to introduce four variants to each one of the eight types of projects defined above. In what follows these variants are denoted as follows: c1s1, c1s2, c2s1, and c2s2. Let us remark that the types of dummy projects previously introduced make no use of the performances that led to define the utility values other than the extreme. Consequently, encodings b1, b2, b3, c3, and s3, have no intervention in the procedure that we followed to assign numerical values to the capacities.

Each variant was presented to the experts using a set of 8 cards (a set per variant). On each card we wrote down the 6 performances that characterized the variant of the considered project (the corresponding utilities were not on the card). The experts were presented with the set of cards associated with the variant c1s1. We explained that they first should rank the cards by a nondecreasing order of the satisfaction level associated with the considered projects. Then, they should insert blank cards to differentiate the extent of the differences that separate these satisfaction levels. Finally, we asked the experts to assign a numerical value (denoted by $z$ ) to the ratio between the satisfaction levels of the projects ranked in the best position and those ranked in the worst position. In a first phase, the experts worked separately, successively for each one of the four variants, then they worked collectively (denoted by expert e4).
The experts accepted the work with the cards with no difficulties. They quickly understood they should express their higher or lower satisfaction with respect to each of the dummy projects as they were characterized on the cards with the purpose of requalifying the abandoned quarry. To the question posed by the analyst: "What do you think about the followed approach?", both experts declared that the use of the cards was very well suited (when they worked separately). They also recognised that: "The possibility of handling the cards led to successive discussions related to their relative positions as well as to the number of blank cards to be inserted into the intervals" and that, "the discussion strongly helped to reach a consensus". The experts specified that in the cases were disagreement persisted regarding the ranking of some pairs of cards, they decided to consider such pairs in the same position. Then, the analyst asked them to compare the ranking finally obtained (expert e4) with the rankings selected when working separately (see Table 4).

After underlining that projects $p_{45}$ and $p_{15}$ always occupied the best positions in the ranking and project $p_{6}$ occupied the worst position (exception being the place of $p_{45}$ by expert e2, who considered $p_{1}$ and $p_{2}$ in better positions than $p_{45}$ since he favoured the profitability and costs; $p_{15}$ was ranked in the first position by this expert since its investment costs are small while it provides good performances in terms of the environmental aspects), the analyst highlighted the most important divergences and asked the experts to explain them. More precisely, expert e1 (expert in economic evaluation) indicated that she favored project $p_{4}$ because she judged it particularly important to improve the landscape ecology of a large naturalized surface. In the focus group work, she realized that the question was a bit different since expert e2 (environmental engineer) explained that the covered surface for the landscape for the naturalized area was not particularly large (at most $6.5 \mathrm{ha}$ ). Expert e1 also drew the attention about the fact that project $p_{1}$ was very cheap, but since it did not produce any effect, ranking it in a good position was not justified. This explanation led e2 to accept the ranking finally obtained. Other interesting discussions took place, particularly regarding project $p_{2}$, but due to space constraints they are not described in this article.

The experts unanimously agreed in underlining that the excessively unrealistic features of some projects made it difficult to define a ranking. Other rankings could, according to them, be justified. These unrealistic features were due to large differences in cost that separated the cheapest projects from the most expensive. They were also due to the scarcity of information that characterized the projects. Finally, regarding the $z$ value, the experts appreciated the possibility offered of expressing the values through an interval, except for expert e1 who had no hesitations in providing a single value for $z$.

On these grounds and by applying the procedure in Section 3.4 several sets of numerical values were assigned to the capacities (see Table 7 in Appendix A).

\section{Computational experiments and results}

This section starts by explaining the design of the experiments, then it introduces two specific sets of threshold functions, and finally, it presents the obtained results.

\subsection{The design of the experiments}

Each computational experiment, which leads to the assignment of a utility value to each one of the five actions, $a_{1}, \ldots, a_{5}$, with the Choquet integral, requires the precise definition of the conditions in which such an experiment has been performed. Therefore, a particular choice of these conditions (or options) constitutes what 
Table 4

Rankings of projects and blank cards by expert.

\begin{tabular}{|c|c|c|c|c|c|c|c|c|c|c|c|c|c|c|c|c|c|c|}
\hline & & \multicolumn{15}{|c|}{ Ranks and blank cards } & \multirow[t]{2}{*}{$z^{\mathrm{min}}$} & \multirow[t]{2}{*}{$z^{\max }$} \\
\hline & & $R_{1}$ & $e_{1}$ & $R_{2}$ & $e_{2}$ & $R_{3}$ & $e_{2}$ & $R_{4}$ & $e_{4}$ & $R_{5}$ & $e_{5}$ & $R_{6}$ & $e_{6}$ & $R_{7}$ & $e_{7}$ & $R_{8}$ & & \\
\hline \multirow[t]{4}{*}{ Expert e1 } & c1s 1 & $p_{6}$ & 0 & $p_{1}$ & 0 & $p_{5}$ & 0 & $p_{2}, p_{3}$ & 0 & $p_{4}$ & 0 & $p_{45}$ & 2 & $p_{15}$ & & & 80 & 80 \\
\hline & $\mathrm{c} 1 \mathrm{~s} 2$ & $p_{6}$ & 0 & $p_{1}$ & 0 & $p_{5}$ & 0 & $p_{2}, p_{3}$ & 0 & $p_{4}$ & 0 & $p_{45}$ & 2 & $p_{15}$ & & & 80 & 80 \\
\hline & $\mathrm{c} 2 \mathrm{~s} 1$ & $p_{6}$ & 0 & $p_{1}$ & 0 & $p_{5}$ & 0 & $p_{2}, p_{3}$ & 0 & $p_{4}$ & 0 & $p_{45}$ & 4 & $p_{15}$ & & & 100 & 100 \\
\hline & $c 2 s 2$ & $p_{6}$ & 0 & $p_{1}$ & 0 & $p_{5}$ & 0 & $p_{2}, p_{3}$ & 0 & $p_{4}$ & 0 & $p_{45}$ & 4 & $p_{15}$ & & & 100 & 100 \\
\hline \multirow[t]{4}{*}{ Expert e2 } & c1s 1 & $p_{6}$ & 1 & $p_{3}, p_{4}, p_{5}$ & 1 & $p_{45}$ & 4 & $p_{1}$ & 2 & $p_{2}$ & 1 & $p_{15}$ & & & & & 70 & 70 \\
\hline & $\mathrm{c} 1 \mathrm{~s} 2$ & $p_{6}$ & 1 & $p_{3}, p_{4}, p_{5}$ & 1 & $p_{45}$ & 4 & $p_{1}$ & 2 & $p_{2}$ & 1 & $p_{15}$ & & & & & 70 & 70 \\
\hline & $\mathrm{c} 2 \mathrm{~s} 1$ & $p_{6}$ & 1 & $p_{3}, p_{4}, p_{5}$ & 1 & $p_{45}$ & 5 & $p_{1}$ & 3 & $p_{2}$ & 1 & $p_{15}$ & & & & & 70 & 70 \\
\hline & $\mathrm{c} 2 \mathrm{~s} 2$ & $p_{6}$ & 1 & $p_{3}, p_{4}, p_{5}$ & 1 & $p_{45}$ & 4 & $p_{1}$ & 4 & $p_{2}$ & 1 & $p_{15}$ & & & & & 70 & 70 \\
\hline \multirow[t]{4}{*}{ Expert e3 } & c1s 1 & $p_{6}$ & 3 & $p_{2}$ & 2 & $p_{1}$ & 2 & $p_{4}$ & 4 & $p_{3}$ & 2 & $p_{5}$ & 3 & $p_{45}$ & 4 & $p_{15}$ & 20 & 25 \\
\hline & $\mathrm{c} 1 \mathrm{~s} 2$ & $p_{6}$ & 3 & $p_{2}$ & 2 & $p_{1}$ & 3 & $p_{4}$ & 3 & $p_{3}$ & 2 & $p_{5}$ & 4 & $p_{45}$ & 2 & $p_{15}$ & 15 & 20 \\
\hline & $\mathrm{c} 2 \mathrm{~s} 1$ & $p_{6}$ & 3 & $p_{2}$ & 3 & $p_{1}$ & 2 & $p_{4}$ & 3 & $p_{3}$ & 2 & $p_{5}$ & 3 & $p_{45}$ & 5 & $p_{15}$ & 20 & 25 \\
\hline & $c 2 s 2$ & $p_{6}$ & 3 & $p_{2}$ & 3 & $p_{1}$ & 3 & $p_{4}$ & 2 & $p_{3}$ & 2 & $p_{5}$ & 5 & $p_{45}$ & 4 & $p_{15}$ & 20 & 30 \\
\hline \multirow[t]{4}{*}{ Expert e4 } & c1s 1 & $p_{6}$ & 3 & $p_{3}$ & 1 & $p_{4}, p_{5}$ & 3 & $p_{1}$ & 4 & $p_{2}, p_{45}$ & 4 & $p_{15}$ & & & & & 90 & 90 \\
\hline & $\mathrm{c} 1 \mathrm{~s} 2$ & $p_{6}$ & 1 & $p_{3}$ & 1 & $p_{4}, p_{5}$ & 3 & $p_{45}$ & 1 & $p_{2}$ & 5 & $p_{1}$ & 3 & $p_{15}$ & & & 90 & 90 \\
\hline & $\mathrm{c} 2 \mathrm{~s} 1$ & $p_{6}$ & 5 & $p_{1}$ & 2 & $p_{3}$ & 2 & $p_{4}, p_{5}$ & 2 & $p_{2}$ & 0 & $p_{45}$ & 5 & $p_{15}$ & & & 90 & 90 \\
\hline & $\mathrm{c} 2 \mathrm{~s} 2$ & $p_{6}$ & 5 & $p_{1}$ & 1 & $p_{3}$ & 3 & $p_{4}, p_{5}$ & 3 & $p_{2}, p_{45}$ & 5 & $p_{15}$ & & & & & 100 & 100 \\
\hline
\end{tabular}

we call a configuration. A configuration is defined by the following elements:

- The way in which the utilities have been assigned to the numerical scale levels of criteria $g_{1}$ (i.e., the levels of costs) and $g_{4}$ (i.e., the levels of surface). For each criterion, three ways of encoding the scale levels have been considered: c1, c2, c3 and s1, s2, s3 (see Section 4).

- The way in which the utilities have been assigned to the verbal scale levels of criteria $g_{2}, g_{3}$, and $g_{5}$. Three ways of encoding the scale levels have been considered: b1, b2, b3 (see Section 4).

- The expert, who, in the considered conditions, has defined the way in which $s$ he ranks the projects, $p_{1}, \ldots, p_{8}$ (see Section 4 ), where $p_{7}=p_{45}$ and $p_{8}=p_{15}$. Four experts have been taken into account: e1, e2, e3, e4.

- The chosen $z$ ratio value (from now on " $z$ " will be used instead) for performing the computations. Two values for $z$ have been considered: the minimum (min) and the maximum (max) values provided by the considered expert.

- The number of blank cards placed in each one of the seven intervals in the ranking of the projects, $p_{1}, \ldots, p_{8}$. Firstly, we started by taking into account the number of blank cards (with their respective position in the ranking) as it has been proposed by the considered expert (from now on this number will be identified as onc - original number of cards). Secondly, for the reasons presented in Sections 5.2 and 5.3, we took into account a modification of the blank cards (from now on this number will be identified as ace - adding one card everywhere, i.e., in every interval).

Thus, every configuration is characterized by a code of the type: $\mathrm{c}(\mathrm{i}) \mathrm{s}(\mathrm{j}) \mathrm{b}(\mathrm{k}) \mathrm{e}(\mathrm{h}) \mathrm{z}(\min , \max )$ onclace, with $i=1,2,3, j=$ $1,2,3, k=1,2,3,4$, and $h=1,2,3,4$ (note that $\mathrm{b} 4=\mathrm{b} 1$ ).

The purpose of the performed experiments is to examine to which extent the results are affected or influenced by the following three aspects: (1) the way of encoding the different numerical and verbal scales levels during the individual and collective work of the experts; (2) the ill-determined value of $z$; and, (3) the number (and place) of the blank cards inserted into the intervals of the ranking of projects, $p_{1}, \ldots, p_{8}$.

At first we have been interested in the results obtained with the 192 configurations characterized as follows: $\mathrm{c}(\mathrm{i}) \mathrm{s}(\mathrm{j}) \mathrm{b}(\mathrm{k}) \mathrm{e}(\mathrm{h}) \mathrm{z}(\min , \max )$ onc and ace, for $i, j=1,2$, $k=1,2,3,4$ and $h=1,2,3,4$.

Starting from the conclusions that we reached in examining the results obtained with these 192 configurations, in a second stage, we considered the encodings $\mathrm{c} 3$ and $\mathrm{s} 3$ along with the new ace encoding. This process was done not by merging the new encodings with the 192 configurations, as analyzed in the first stage, but only with some (well defined) of them. The purpose of this second phase was to confirm or to challenge the results obtained in the first stage of the study based on the examination of the 192 configurations.

Before presenting the sets of results obtained (see Section 5.3), it is necessary to clarify the way we proceeded to judge if the utility values assigned to the actions, $a_{1}, \ldots, a_{5}$, in two different configurations should be seen as significantly different. For such a purpose, it is necessary to check to what extent the utility value of a given action could be influenced in a non significant way by some difficulties and hesitations experienced by the expert during the decision aiding process (see Section 4). It is important to recall that this process led herlhim to build the required data necessary to assign a utility value to each one of the five actions by using the Choquet integral. These data relate to both the ranking of the projects, $p_{1}, \ldots, p_{8}$, and the value assigned to $z$. The purpose of the next subsection is to show how we have addressed this task by building two specific sets of threshold functions.

\subsection{Two sets of threshold functions}

The analyses of the impact of both the ill-determination of $z$ and the number and position of blank cards led us to build two sets of threshold functions. Regarding the ill-determination of the $z$ value, we studied the relationship, through a linear regression analysis technique, between the differences, in absolute value, of the utility for a maximum and a minimum value of this parameter and the minimum value of the utility. We observed that the differences vary with the minimum utility value, which led us to construct variable (indifference and preference) thresholds functions. The same kind of analyses were performed for the illdetermination of the number and positions of the blank cards and similar results were obtained. These analyses are presented in the next three subsections.

The next subsection proposes a detailed explanation of how to construct the two sets of threshold functions. The reader interested directly into the results may wish to move to Section 5.3. 


\subsubsection{Analyzing the impact of the ill-determination of $z$}

In order to analyze the influence of the ill-determination of $z$, it was necessary to compare the values of the utilities assigned to each one of the five actions in the pairs of configurations characterized in the same way, but for the $z$ value. To accomplish this task we started by taking the pairs of the obtained results as follows: $\mathrm{c}(\mathrm{i}) \mathrm{s}(\mathrm{j}) \mathrm{b}(\mathrm{k}) \mathrm{e}(\mathrm{h})$ onc with $i, j=1,2, k=1,2,3,4$, and $h=1,2,3,4$. Each pair is formed by the results obtained with zmin and zmax. There are 48 pairs of possible configurations. We focused our analysis on the ones which associate:

- With each one of the experts e ( $h)(h=1,2,3,4)$, the encoding $\mathrm{b}(\mathrm{k})(k=1,2,3,4) \mathrm{s}$ he has defined to assign a utility to each level of the verbal scale (see Section 4).

- With the expert e4, each one of the three encodings $\mathrm{b} 1=\mathrm{b} 4, \mathrm{~b} 2, \mathrm{~b} 3$.

- With the experts e2 and e3, the uniform encoding b1, which has been considered relevant by them.

There are thus 32 pairs of configurations. It is also necessary to draw the attention of the reader to the fact that some pairs (more precisely 8 of them) did not allow to assign a utility to the five actions. This impossibility was related to the cases where the way in which the expert ranked the projects, $p_{1}, \ldots, p_{8}$, by inserting blank cards in the intervals proved to be non conform to the hypotheses established regarding the interaction between criteria (see Section 2). This non conformity occurs because one of the consistency conditions as defined in expressions (8) or (9) is not fulfilled by the values assigned to the $\mu$ coefficients of the Choquet integral. These pairs, which led to a particular analysis (see Section 5.3.5 ), are not considered in the current section.

According to the definition of the discriminating threshold functions (see Roy, Figueira, \& Almeida-Dias, 2014) the set of functions to be built aims at understanding the part of arbitrariness, due to the ill-determination of $z$, which affects the utility values assigned to the actions by the Choquet integral. This arbitrariness is highlighted by the differences that separate, for each action, these utilities when they are assigned under identical conditions, but with different $\mathrm{z}$ values. These values, which are $32-8=24$ for each action $a_{i}$, are denoted by $u^{z m i n}\left(a_{i}\right)$ and $u^{z \max }\left(a_{i}\right)$. In order to build direct threshold functions, we have to look at the way the $5 \times 24=120$ differences of utility in absolute value, $\left|u^{z \min }\left(a_{i}\right)-u^{z \max }\left(a_{i}\right)\right|=\Delta^{z}\left(a_{i}\right)$, vary with respect to the smallest of the two values, i.e., $\min \left\{u^{z \min }\left(a_{i}\right), u^{z \max }\left(a_{i}\right)\right\}=\min ^{z}$ $\left\{a_{i}\right\}$ (see Fig. 1 in Appendix C).

Surprisingly, we observed that the hypothesis of direct thresholds, i.e., thresholds that are independent from the utility values in the abscissa axis, does not seem to be acceptable. The regression line (see Fig. 1) confirms this observation. This line highlights the fact (which is clearly visible in the graph) that, in most cases, when the utility values increase, the absolute values of the differences tend to decrease. This led us to conclude that there was a need to build affine threshold functions. With the aim of constructing such functions, we observed the dispersion of the differences. This distribution is presented in Table 8 in Appendix B (where $N b$. is the number of cases, and the repeated values are in between parenthesis). The examination of this table allows to state that:

- 102 of the smallest values of $\Delta^{z}\left(a_{i}\right)$ are at most equal to 0.0029 . - The interval $[0.003,0.0065]$ does not contain any value for $\Delta^{z}\left(a_{i}\right)$, and the same occurs for the interval [0.0085, 0.0109].

- 7 of the 120 values of $\Delta^{z}\left(a_{i}\right)$ belong to the interval [0.0066, $0.0084]$

- $\Delta^{z}\left(a_{i}\right)$ is greater than or equal to 0.0011 for 9 of the 120 values.

Let us consider two actions, $a_{i}$ and $a_{j}$, for which the Choquet integral assigns two values (in the same configuration) such that $u\left(a_{i}\right)<u\left(a_{j}\right)$. When these utilities correspond to those observed in one of the 101 first cases mentioned above, we assumed that the ill-determined knowledge of the $\mathrm{z}$ value that was considered to define these utility values, lead to a difference $\Delta^{z}\left(a_{i}\right)$ that is not significant of a preference. Moreover, we considered that only in the last 9 cases it would be possible to have a strict preference of $a_{j}$ compared with $a_{i}$. On these bases, we put: $q^{z}\left(u\left(a_{i}\right)\right)=0.006$ for $u\left(a_{i}\right)=0.250$, and $q^{z}\left(u\left(a_{i}\right)\right)=0.004$ for $u\left(a_{i}\right)=0.500$. This led to the following indifference threshold function:

$q^{z}\left(u\left(a_{i}\right)\right)=-0.008 u\left(a_{i}\right)+0.008$.

It is easy to check that with this function there is indeed indif-ference for the first 101 values of Table 8 and preference for the remaining ones.

Certainly, this function is not the only one that allows to reach this objective. However, given the use that is made of this threshold function (see Section 5.2.3), the reader will understand with no difficulties, that if this function was replaced by other threshold functions leading to the same cases of indifference and preference, it could not significantly affect the subsequent conclusions resulting from the choice of the threshold function we considered.

To define a preference threshold function able to take into account the above referred objective, we consider: $p^{z}\left(u\left(a_{i}\right)\right)=0.011$ for $u\left(a_{i}\right)=0$. 375, and $p^{z}\left(u\left(a_{i}\right)\right)=0.009$ for $u\left(a_{i}\right)=0$. 500. This led to the following indifference threshold function:

$$
p^{z}\left(u\left(a_{i}\right)\right)=-0.016 u\left(a_{i}\right)+0.017 .
$$

This function also allows to achieve the objective. Here again we can notice that this function is not the only one, but what has been said for the choice of the indifference threshold function also applies for the selection of a preference threshold function.

\subsubsection{Analyzing the impact of the ill-determination of the number and position of the blank cards}

In order to analyze this impact, we need to compare the values of the utilities assigned to each one of the five actions in the pairs of configurations, which were characterized in the same way, but for the number and location of blank cards in the ranking of projects, $p_{1}, \ldots, p_{8}$. It is, however, necessary that the two dispositions of these blank cards are close enough to reflect the difficulty of the expert when $s \backslash$ he makes the decision about the number of blank cards to be inserted into each one of the seven intervals. This led us to introduce a first modification, denoted by ace, from the number and initial disposition onc of the blank cards as they were inserted by the expert in the seven intervals of the projects ranking. The configuration ace is deduced from onc by adding a blank card in each one of the seven intervals.

In order to make possible the comparison, we started by taking the pairs of the obtained results as follows: $\mathrm{c}(\mathrm{i}) \mathrm{s}(\mathrm{j}) \mathrm{b}(\mathrm{k}) \mathrm{e}(\mathrm{h}) \mathrm{zmin}$ with $i, j=1,2, \quad k=1,2,3, \quad$ and $h=1,2,3,4$.

Each pair is formed by the results obtained with the configurations onc and ace. Only the 24 of the 48 pairs taken into account in Section 5.2.1 were again considered here (for the same reasons). Therefore, for each action $a_{i}$, we obtained 24 pairs denoted by $u^{\text {onc }}\left(a_{i}\right)$ and $u^{\text {ace }}\left(a_{i}\right)$. Here again we can observe that, the greater the smallest of these two values, $\min ^{b c}\left\{a_{i}\right\}$, the more the absolute value, $\left|u^{\text {onc }}\left(a_{i}\right)-u^{a c e}\left(a_{i}\right)\right|=\Delta^{b c}\left(a_{i}\right)$, tends to diminish (see Fig. 2 in Appendix C. Thus, it is necessary, as before, to build affine threshold functions. For such a purpose, we looked at the repartition of the new $\Delta^{b c}\left(a_{i}\right)$ values (see Table 9 in Appendix B. The examination of this table allows to conclude that:

- 104 of the smallest values of $\Delta^{b c}\left(a_{i}\right)$ are at most equal to 0.0148 .

- The interval $[0.0149,0.0171]$ does not contain any value for $\Delta^{b c}\left(a_{i}\right)$, and the same occurs for the interval [0.0249, 0.0316]. 
- 12 of the 120 values of $\Delta^{b c}\left(a_{i}\right)$ belong to the interval [0.0172, 0.0248].

- $\Delta^{b c}\left(a_{i}\right)$ is greater than or equal to 0.0317 for 4 of the 120 values.

When the Choquet integral assigns (in the same configuration) to two actions the utilities that correspond to those utilities observed in the first above 104 cases, we considered that the illdetermination of the number and position of the blank cards, which have been taken into account to assign these values, lead to a difference $\Delta^{b c}\left(a_{i}\right)$ that is not significant of a preference, and only for the last 4 cases it would be possible to have a strict preference of one of the actions with respect to the other. On these bases, we consider: $q^{b c}\left(u\left(a_{i}\right)\right)=0.017$ for $u\left(a_{i}\right)=0.250$, and $q^{b c}\left(u\left(a_{i}\right)\right)=0.014$ for $u\left(a_{i}\right)=0.400$. This led to the following indifference threshold function:

$q^{b c}\left(u\left(a_{i}\right)\right)=-0.020 u\left(a_{i}\right)+0.022$.

Analogously, we consider: $q^{b c}\left(u\left(a_{i}\right)\right)=0.028$ for $u\left(a_{i}\right)=0.250$ and $q^{b c}\left(u\left(a_{i}\right)\right)=0.020$ for $u\left(a_{i}\right)=0$. 400. This led to the following preference threshold function:

$p^{b c}\left(u\left(a_{i}\right)\right)=-0.032 u\left(a_{i}\right)+0.036$.

The reader can also check that with this set of threshold function the objective is achieved.

\subsubsection{On the way the two threshold functions have been used}

In this article the Choquet integral was used to assign utility values to each one of the five actions, $a_{1}, \ldots, a_{5}$, with the purpose of making possible their comparison. The implementation of the Choquet integral was developed according to two subsequent steps. Firstly, it was necessary to assign utilities to the different criteria scale levels. In this first step several options are available (see Section 3). In a second step, the intervention of an expert (see Section 4) became necessary to assign numbers to the $\mu$ parameters of the Choquet integral. Our purpose is to highlight the influence that the choice of the options, which is considered in the first step, and the choice of the expert, which is considered in the second step, may have on the way the obtained utility values by the Choquet integral lead to the comparison of the actions among them. We can perform this task by simply comparing the way the different analyzed configurations rank the actions in a complete order by a decreasing order of their utilities. This way leads to highlight that the non significant differences come only from the two ill-determination factors that have been analyzed in the previous two subsections. It is consequently necessary to take into account the two sets of threshold functions to highlight only the significant differences. With such a purpose in mind, for each one of the complete orders taken into account, we considered a pseudo order preference structure (Roy \& Vincke, 1984), which assigns, for every pair of actions, the most relevant of the following three binary relations: indifference $(I)$, weak preference $(Q)$, which means hesitation between indifference and preference, and strict preference $(P)$, that is preference without any hesitation.

For a given configuration, the two sets of threshold function do not necessarily provide the same pseudo order. Always having in mind the necessary relation of avoiding non significant differences, we only chose to consider the indifference or the preference when there is unanimity with the two sets of functions in favor of one or the other action. Fig. 3 (see Appendix C) shows that it is impossible that one of two functions leads to an indifference when the other leads to a strict preference. This figure also shows that:

- There is indifference with the two sets of threshold functions if and only if there is indifference for $q^{z}\left(u\left(a_{i}\right)\right)$.

- There is strict preference with the two sets of threshold functions if and only if there is preference for $p^{b c}\left(u\left(a_{i}\right)\right)$.
These elements led us to associate with each configuration a unique pseudo order.

\subsection{Presentation of the obtained results}

After a brief introduction, this section presents the analyses of the influence or impact on the results of the following elements: the experts, the chosen encodings for the cost and surface criteria, and the number and position of the blank cards.

The next paragraph provides interesting insights about the developed study. With respect to the impact on the results of the different experts, some conclusions can be summarized as follows: action $a_{2}$ is always ranked in the first position and there is no configuration for which the four experts rank the other four actions in the same way. We can also mention that in each configuration there is at least one expert that ranks in position 2 an action, which is ranked in the last position by another expert. Concerning the impact of the encodings on the results, we observed that significant effects may occur mainly with the way of encoding the numerical scales. The changes in the number and the position of the blank cards can also produce some significant impacts on the results.

\subsubsection{Preliminaries}

As we have explained at the beginning of Section 5.1, the application of the Choquet integral for assigning a utility value to each one of the five actions, $a_{1}, \ldots, a_{5}$, needs the selection of one option, for each one of the considered characteristics. This leads to the definition of what we called a configuration. This is done with the purpose of establishing the conditions under which the computations are performed. It is also important to recall that the purpose of this paper is to highlight the less or more significant impact related to the choice of the way the actions under analysis can be ranked.

Consider two configurations, which are only different in a single option selected taken into account one of the characteristics. We say that the choice between one or the other of these two options is with no impact in this configuration if the resulting pseudo orders in one and the other case are identical. We state that such a choice is with no significant impact when it is with no impact in a set of configurations judged representative. This set may contain only a small number of configurations for which the pseudo orders are not rigorously identical, but they are only different for the replacement of a weak preference either by an indifference or by a strict preference.

The obtained results allow to state that the choice between the options zmin and zmax is with no significant impact. For such a reason the results presented in the next subsections are all related to the option zmin.

Let us also recall that three encodings were considered to obtain the utility values for the verbal scale levels. More precisely, these encodings are the uniform encoding $b 1$ of expert $e 1$, the encoding b2 of expert e2, and the encoding b3 of expert e3 (see Section 4). The obtained results show that in many configurations the choice of the encoding is with no impact. However, in some of them the obtained pseudo orders may differ not only for the replacement of a weak preference either by an indifference or by a strict preference, but also for the replacement of an indifference by a strict preference and even by a reversal in the order in which the two consecutive actions are ranked. Thus, it is not anymore possible to consider that the way of assigning utility values to the verbal scale levels is with no significant impact. On the one hand, the impact of this choice remains low. For this reason we will not systematically analyze it in the following subsections, while we will only mention some occurrences. On the other hand, the obtained results show that the resulting pseudo orders have big changes according 
to the considered expert (see Section 5.3.2) and also according to the way of assigning utility values to criteria $g_{1}$ (cost) and $g_{4}$ (sur- face) (see Section 5.3.3 ). Section 5.3.4 is devoted to the examina- tion of the impact on the pseudo orders of the number and po- sition of the blank cards in the ranking of projects, $p$ $1, \ldots, p_{8}$. As we have mentioned in Section 5.1, for some configurations it is not possible to assign utility values to the actions since the way the ex- pert ranked the projects, $p_{1}, \ldots$, $p_{8}$, and inserting blank cards into the intervals, has proved to be inconsistent (or non conform) with the interaction between criteria. Section 5.3.5 is thus devoted to the examination of the non conformity cases.

\subsubsection{Analyzing the influence of the experts}

In order to highlight this influence or impact of the ex- perts, we took into account the following set of configurations:

$\mathrm{c}(\mathrm{i}) \mathrm{s}(\mathrm{j}) \mathrm{b}(\mathrm{h}) \mathrm{e}(\mathrm{h}) \mathrm{z}$ (min) onc and ace, for $i, j=1,2$ and the considered expert, $h$, with her $\backslash$ his encoding of blank cards, $\mathrm{h}$ (remember that $\mathrm{b} 4=\mathrm{b} 1$ ). This led to the construction of 8 configurations per expert. For each configuration, Table 10 in Appendix B shows the obtained results for each expert. For the sake of simplicity, in this table, we considered the transitivity of $P$ for non successive pairs; for example, the situation in which $a Q b, a P c$, and $b P c$ is represented by $a Q b P c$.

On the one hand, let us observe that in each of the 8 configurations, when there is conformity, action $a_{2}$ is always placed in rank 1 . On the other hand, there is no configuration for which the 4 experts rank in the same way the other 4 actions. Moreover, in each configuration there is at least one expert that ranks in position 2 an action, which is ranked in the last position by another expert. For example, this happens in the first 6 configurations, between experts e1 and e2, with reference to action $a_{1}$. The same happens for the configuration $\mathrm{c} 2 \mathrm{~s} 2$, between experts e4 and e3, with reference to action $a_{5}$. In rank 2, we find 6 times $a$ 1 , 4 times $a_{5}, 7$ times $a_{4}$, and 4 times $a_{3}$, when in the last position of the rank we find 12 times $a_{1}, 8$ times $a_{3}$, and 6 times $a_{5}$.

In total, with these 32 configurations we obtained 6 non con- formity cases and 13 different pseudo orders: 3 for each one of the experts, e1, e3, e4, and 5 for the expert e2 (for a total of 14 pseudo orders). Let us remark that there is only one common pseudo order for two experts: this is the pseudo order in configuration c1s2ace for e4 and in configuration c2s2onc for e1.

The computations performed for each expert with the reference set took into account, for each one of them, the way the verbal scale has been encoded. We also performed other computations

with the purpose of examining the impact the choice of the encoding could have on the obtained pseudo orders. For this purpose, we started to perform the computations by replacing the encoding

b1 by the encodings b2 and b3, for the experts e2 and e3, respectively. This choice was motivated by the fact that the encoding b1 considered by the expert $\mathrm{e} 1$ and adopted by the expert e 4 (representing the group of the three experts) may be seen as a reference encoding, since it consists of assigning equal variations of utility between two consecutive scale levels, whatever their position in the scale. The obtained results highlight the following aspects:

a) For expert e2, as well as for expert e3, most of the modifications (which are in a large number) consist of replacing a weak preference either by an indifference or by a strict preference. These modifications represent the situations that we considered (see Section 5.3.1) with impacts that deserve to be judged as non significative.

b) For expert e2, moving from b2 to b1 only highlights a significant modification: with the c1s1ace configuration (see Table 10) action $a_{1}$, which was in position 2 , moves to rank
3 , and action $a_{4}$, which was in position 3 , moves to position 2 (with a strict preference).

c) For expert e3, moving from b3 to b1 does not highlight any significant modification.

We then examined, for expert e4, which impact the fact of replacing either b2 or b3 by b1 could have. We also observed that several little significant modifications occur. The replacement of $\mathrm{b} 2$ by $\mathrm{b} 1$ in the $\mathrm{c} 1 \mathrm{~s} 2$ onc configuration (see Table 10) leads to sub- stitute an indifference between $a_{5}$ and $a_{3}$ by a strict preference in favor of $a_{5}$ with respect to $a_{3}$.

Let us observe that the encodings $b 1, b 2$, and $b 3$ associate the 0 and 1 utilities with the same (extreme and median) scale levels. The differences are only related with the other (intermediate) scale levels. In order to highlight the advantage of the little significant impacts, it is undoubtedly necessary to perform some computations with other encodings than the ones with 0 and 1 utility values for the extreme scale levels. We thought it may not be necessary to undertake such a type of calculation for these scales because they appeared to us to be too arbitrary.

\subsubsection{Analyzing the influence of choosing encodings for the cost $\left(\mathrm{g}_{1}\right)$} and the surface $\left(\mathrm{g}_{4}\right)$ criteria scales

For this analysis we took into account the reference set of configurations, after completing it with the following two configurations: $\mathrm{c} 3 \mathrm{~s} 3 \mathrm{~b}(\mathrm{~h}) \mathrm{e}(\mathrm{h})$ zminonc and ace, $h=1,2,3,4$.

Table 11 in Appendix B shows for each expert the obtained pseudo orders along with the configurations which led to obtain such pseudo orders. This table highlights that in many configurations the way of assigning utility values to the numerical scale levels, that were used to characterize the performances of the actions on criteria $g_{1}$ and $g_{4}$, significatively affects the obtained pseudo order for each one of the four experts. Let us start by considering the encodings $c 1$ and $c 2$. They differ from the costs considered for the characterization of the 0 and 1 utilities, respectively. In these two scales, the utility value assigned to a performance $g_{j}$ in a given interval $\left(g_{j}^{\ell}<g_{j}<g_{j}^{u}\right.$ ) is defined in the same way by linear inter- polation (see Eq. (11)).

We observed that, all other things being equal, when the expert works with $c 1$ or $c 2$, the obtained results may differ. It is, for example, the case (see Table 11 ):

- For experts e1, e2, and e4 with the configurations c1s2onc and c2s2onc.

- For expert e3; in this case, it was possible to define a pseudo order, with configurations $\mathrm{c} 2 \mathrm{~s} 2 \mathrm{onc}$ and ace, while this did not happen either with c1s2onc nor with c1s2ace.

Let us consider now the encodings $\mathrm{s} 1$ and $\mathrm{s} 2$ for which the differences are analogous to the ones that differentiate $c 1$ from $c 2$. Also in this case we observed that the results may be significantly different when the expert works with $s 1$ or $s 2$ (all other things being equal). It is, for example, the case:

- For experts e1, e2, and e3, with the configurations c2s1onc and c2s2onc.

- For expert e4; in this case, it was not possible to define a pseudo order with configurations c1s1onc and ace, while this did not happen with the configurations c1s2onc and ace.

The performances that were selected as references for defining the 0 and 1 utility values are the same as in $c 2$ and $c 3$, on one side, and as in s2 and s3, on the other side. Moreover, while in c2 and in $s 2$ the intermediate utility values were defined by linear interpolation (as it was recalled below), in c $3 s 3$ the intermediate utility values were obtained from the interaction with the group of experts, e4. It is the only change that originated the differences 
(where they exist) between the pseudo orders found with the configurations c2s2 (onc, ace), on one side, and the pseudo orders found with the configurations $c 3 s 3$ (onc, ace), on the other side. The examination of Table 11 highlights only two significant modifications:

- With expert e1, the movement from c2s2onc to c3s3onc leads to a change from $a_{4} P\left(a_{3} I a_{5}\right)$ into $a_{4} I a_{5} Q a_{5}$.

- With expert e3, the movement from c2s2onc to c3s3onc leads to a change from $a_{4} P a_{3}$ into $a_{3} Q a_{4}$.

In conclusion, with these 10 configurations we now observe 5 different pseudo orders for e1, 6 for e2, 3 for e3, and 3 for e4 (in total 17). The consideration of the two additional configurations, c3s3onc and ace, highlights 3 new pseudo orders (16 among the 17 are different).

\subsubsection{Analyzing the influence of the number and position of the blank cards}

The difference of the utility values assigned to the actions when (all other things being equal) we added (in a uniform way) a blank card into the intervals of the ranking of projects, $p_{1}, \ldots, p_{8}$, was used to define the second of the two sets of threshold functions.

It is important to start by examining the influence such a uniform change (moving from onc to ace) may have on the obtained pseudo orders. The examination of Table 11 shows that:

- The pseudo orders obtained for two configurations that are only different for the option (onc, ace) are frequently different, but in the majority of the cases there is only a replacement of a weak preference either by an indifference or by a strict pref- erence.

- There are only two significant differences, one with expert e1 and the other with the expert e3 (see in Table 11 the configurations, c2s2b1e1z80onc and c2s2b3e3z20onc, respectively).

Taking into account the way the above mentioned set of threshold functions has been defined, it appeared to us interesting to examine the impact the ill-determination of the number of blank cards could have on one and only one of the intervals of the ranking of projects, $p_{1}, \ldots, p_{8}$. Would such a hesitation of the expert leave the pseudo order unchanged? If there were cases where it was otherwise, would the changes remain non significant? It appeared to us important to examine these two questions for expert e4 (group of the experts). Six configurations are concerned: $c 1 s 2, c 2 s 2, c 3 s 3$ combined with onc and ace (the configurations $\mathrm{c} 1 \mathrm{~s} 2$ and $\mathrm{c} 2 \mathrm{~s} 2$ provide non conform results, see Section 5.3.5).

Let us consider two projects, $p_{x}$ and $p_{y}$, which are in the same position or in consecutive positions in the ranking. In the case where they are consecutive (in that order), adding a supplementary blank card, requires the examination of two new options (denoted in an obvious way) oncpx1py and acepx1py. In the case where $p_{x}$ and $p_{y}$ are in the same position, it is necessary to make a separation of both, i.e., to create an interval between these two projects (which does not necessarily require the introduction of a blank card between $p_{x}$ and $p_{y}$ ). There are two ways of making this separation: ranking them in the order $p_{x} p_{y}$ or in the reverse order $p_{y} p_{x}$. Consequently, there are $2 \times 2=4$ new options, denoted oncpx0py, acepx0py, oncpx1py, and acepy1px.

We have actually seen that the addition of a single blank card often left the pseudo order either unchanged or with non significant modifications. The change is, however, significant in the following configurations:

- c1s2oncp61p1, the pseudo order becomes $a_{2} P\left(a_{4} I a_{3}\right) Q a_{5} P a_{1}$ instead of $a_{2} P a_{4} P\left(a_{5} I a_{3}\right) P a_{1}$.
- c1s2oncp31p4, the pseudo order becomes $a_{2} P a_{4} Q a_{5} Q a_{3} P a_{1}$ instead of $a_{2} P a_{4} P\left(a_{5} I a_{3}\right) P a_{1}$.

- c1s2oncp40p5, the pseudo order becomes $a_{2} P a_{4} P a_{5} P a_{3} P a_{1}$ instead of $a_{2} P a_{4} P\left(a_{5} I a_{3}\right) P a_{1}$.

- c3s3oncp71p2, the pseudo order becomes $a_{2} P a_{4} P a_{3} P a_{1} P a_{5}$ instead of $a_{2} P a_{5} P a_{4} P a_{3} P a_{1}$.

- c3s3acep71p2, the same modification as in the previous configuration.

Taking into account these 5 significant results, we decided it would be useless to do the same kind of analysis for the experts, e1, e2, and e3. We observed that the experts frequently hesitate on the number of blank cards to be inserted not only into a single interval of the ranking of projects, $p_{1}, \ldots, p_{8}$, but also into several intervals. Thus, there is a strong indication that in order to take into account the effect of such conjoint hesitations, we can only increase the number of cases in which the obtained pseudo orders are significantly different.

It should be noted that the following pseudo orders are new (i.e., they are not in Table 11): $a_{2} P\left(a_{4} I a_{3}\right) Q a_{5} P a_{1}, a_{2} P a_{4} Q a_{5} Q a_{3} P a$ 1, and $a_{2} P a_{4} P a_{5} P a_{3} P a_{1}$.

\subsubsection{Analyzing the cases of non conformity}

The non conformity (i.e., the violation of the mutualstrengthening and $\backslash$ or mutual-weakening conditions) has been observed in the following cases (see Table 11):

- With expert e3, the mutual-strengthening condition is violated for configurations c1s2onc and c1s2ace.

- With expert e4, the mutual-weakening condition is violated for configurations c1s1onc and c1s1ace, and the mutualstrengthening condition is violated for configurations $\mathrm{c} 2 \mathrm{~s} 1 \mathrm{onc}$ and c2s1ace.

We have started by finding the cases for which it was possible to restore the conformity either by separating two projects in the same position or by adding one more blank card into one of the intervals of the ranking of projects, $p_{1}, \ldots, p_{8}$. The only possible cases are the following:

- With expert e3, the only configuration that permits the restoration of the mutual-strengthening condition is c1s 2 oncp71p8; we obtain thus the pseudo order $a_{2} P_{3} P a_{4} P a_{1} P a_{5}$.

- With expert $\mathrm{e} 4$, the only configuration that permits the restoration of the mutual-strengthening condition is c2s1oncp $11 \mathrm{p} 8$; we obtain thus the pseudo order $a_{2} \mathrm{~Pa}_{4} \mathrm{~Pa}_{1} \mathrm{Qa}_{3} \mathrm{~Pa}_{5}$.

In the remaining cases of non conform configurations, we investigated the minimum number of modifications to be performed in order to restore the conformity. The results are as follows:

- With expert e3, only a single configuration is involved, i.e., c1s 2 ace. In this case we needed to add two blank cards between $p_{7}$ and $p_{8}$ to restore the conformity; the obtained pseudo order was the same as in the above configuration c1s2onc.

- With expert e4, three configurations were involved:

i) For configurations c1s1onc and ace the mutualweakening condition was violated (we can recall that the projects were ranked as follows: $p_{6} 3 p_{3} 1\left(p_{4}, p_{5}\right) 3 p_{1} 4\left(p_{2}\right.$, $\left.\left.p_{7}\right) 4 p_{8}\right)$. We started by examining if it was possible to avoid the violation of the mutual-weakening condition by placing $p_{7}$ before $p_{2}$ and by adding a high enough number of blank cards between them: unfortunately, this was impossible. The final solution found was the following: To place $p_{1}$ and $p_{7}$ is the same position and insert a blank card between these two projects and $p_{2}$ (when adding a single blank card the mutual-weakening condition is no longer violated, but the mutual-strengthening condition becomes violated). 
In this case the pseudo order found is the following: $a_{2} P a_{4} P\left(a_{5} I a_{1}\right) P a_{3}$.

ii) For configuration $\mathrm{c} 2 \mathrm{~s} 1 \mathrm{ace}$ the mutual-strengthening condition was violated. In order to restore the conformity it is now necessary to add two blank cards in between $p_{1}$ and $p_{8}$, and not only a single blank card as it was in the case of the configuration $\mathrm{c} 2 \mathrm{~s} 1 \mathrm{onc}$. The ranking remains the same as in the previous case.

It should be noted that the following pseudo orders are new (i.e., they are not in Table 11): $a_{2} P a_{4} P a_{1} Q a_{3} P a_{5}$ and $a_{2} P a_{4} P\left(a_{5} I a_{1}\right) P a_{3}$.

\section{Conclusions}

The results presented in Section 5 lead to the following conclusions:

a) The application of the Choquet integral to justify a ranking of the five actions, $a_{1}, \ldots, a_{5}$, based on the work performed by the experts, leads to the identification of $a_{2}$ as being the sig- nificatively better ranked action, and this happened for all the experts and the data considered for the implementation of the Choquet integral. However, regarding the other four actions, only very weak or partial conclusions (which differ from the experts and the considered configurations) can be drawn (see Table 11 ). It is not surprising that experts e1, e2, and e3 (who have been working separately) obtain different results. They do not necessarily assess the role of each criterion and the inten- sity of the interactions in the same way. This is why we de- cided to make them work together (expert e4 ). The experience exchange that took place (exchange that the experts found to be constructive - see Section 4 ), have resulted in a consensus. With the resulting data of this consensus, the Choquet integral does provide a ranking of the actions for 6 of the 10 config- urations studied. Three rankings were obtained: In the second position, behind $a$ 2 , we have either $a_{4}$ or $a_{5}$; action $a_{1}$ always occupies the last position; and, $a_{3}$ always occupies the last but one position. However, the position of $a_{3}$ appears to be very sensitive to the number of blank cards (see Section 5.3.4 ). Indeed, it is sufficient to add a single blank card in certain intervals to rank $a_{3}$ either in the third position or even in the second position tied with $a_{4}$. Finally, in the 4 non-conform configurations, it may be enough to add one blank card in one and only one interval of the ranking of projects, $p_{1} \ldots, p$ 8 , to restore the conformity. This can lead to having $a_{5}$ in the last position instead of $a_{1}$, which moves to the third position (see Section 5.3.5 ).

b) This application of the Choquet integral with four experts highlights the following two points.

(1) The way the initial performances have been transformed to be encoded on a common utility scale:

- has a non significant influence on the results when the initial performances are modeled in a verbal way on a seven-level scale (from "very bad" to "very good") since we established that the levels "very bad", "average", and "very good" should naturally be encoded on the util- ity scale with the values $0,0.5$, and 1 , respectively (see Section 5.3.1).

- has a significant influence on the results when the initial performances are modeled on a numerical scale that refers to costs and surfaces (see Section 5.3.3 ). With these scales the choice of the extreme values, which were used as reference levels to encode the 0 and 1 utility values, are more arbitrary. It is thus the choice of such performances that has the most signifi- cant influence on the results. The way the intermediate performances are encoded may also have an impact, but in a less systematic way.

(2) The experts worked together to assign numerical values to the capacities of the Choquet integral; this has shown that:

- The choice of a value for the ratio $z$ within the indetermination interval defined by the experts has a non significant impact on the results (see Section 5.3.1).

- The way the projects, $p_{1}, \ldots, p_{8}$, were ranked as well as the number of blank cards inserted in the intervals has, in most of the studied configurations, a significant impact on the results (see Sections 5.3.4 and 5.3.5 ).

c) The experts' reactions during the working phase that was performed to build the required data for the implementation of the Choquet integral, led us to the following findings:

(1) The deck of cards method that was presented to assign utility values to the verbal scale levels was quickly understood by each of the experts, who subsequently have used it with no difficulties (see Section 4.1 ). As for the numerical scales the approach that was followed to define the encodings c3 and s3 (see Section 4.2) did not lead to any difficulty.

(2) The proposed approach regarding the assignment of numerical values to the capacities highlighted some difficulties (see Section 4.3 ). This leads us to call the attention of the reader to the following aspects:

- Concerning the assignment of numerical values to the ratio $z$, the experts found this exercise quite difficult and they appreciated the possibility of defining only one range for the value of this ratio.

- When the experts worked separately, they frequently hesitated about adopting a ranking of the eight projects for each of the four families proposed to them. In their opinion, what often made the comparison less significant was, on the one hand, the unrealistic nature of the projects and, on the other hand, the large differences of their performances on incommensurable scales. Moreover, after adopting a ranking, the number of blank cards to be inserted in each of the intervals could again be a source of hesitation.

- In the group work (expert e4), the experts appreciated the method. They also appreciated the work with the blank cards and considered it a good basis for conducting a decision aiding process with multiple actors. They argued that this way of working allows everyone to clearly explain the way in which they wish to rank the projects and to insert more or less blank cards in the intervals. Moreover, the ability to handle the cards during the discussion was considered appropriate to reach a consensus.

ci) We highlight that we worked with the same experts and the same data used for the study on the ELECTRE III method with interaction between criteria (Bottero et al., 2015). In the previous work, the results obtained were validated by the experts, namely: project $a_{2}$ was the first in the ranking before $a_{3}$, which occupied the second position. These two projects being clearly separated from the other three. It should be mentioned that this special position of $a_{3}$ did not come from the fact that in ELECTRE III the antagonism effect had been taken into account (note that this effect cannot be considered with the Choquet integral). Although the purpose of this article is not to compare the results obtained with two different methods, we may wish to understand why with the Choquet integral the same project does not always appear in second place (as a legitimate second), behind $a_{2}$. It seems to us (but it should be supported by other experiments), that we can present the following argument. The arbitrariness that is inevitably present in the construction of the required data to justify a ranking is less 
well controlled in the Choquet integral than in the ELECTRE III method with interaction between criteria. In the example studied, if we consider the case of $a_{3}$ (see Table 11, the results were highlighted in Sections 5.3.4 and 5.3.5) we observe that the position of $a_{3}$ varies in particular due to the following two factors:

- The choice of the extreme performances for criteria $g_{1}$ and $g_{4}$ which should serve to characterize the 0 and 1 values of the common utility scale.

- The choice of a ranking of the reference (not realistic) projects generates large differences in performances on scales that are difficult to commensurate. Note that such a ranking is used to take into account the interaction effects (mutual-strengthening and mutual-weakening).

The use of the Choquet integral to justify a ranking requires the examination of the impact of the arbitrariness that is present in the two preceding choices. Further work will seek to:

- Encode in terms of utility the performances of the numerical scales in a different way by taking into account the extreme performances of these scales, for example, in verbal terms.

- To define the projects to be ranked in order to obtain the numerical values of the capacities in a more realistic way by avoiding the presence of the extreme performances. Furthermore, we can envisage taking into account more reference projects than those related with the criteria and to the pairs of interacting criteria considered in the Choquet integral approach we have proposed, in order to mitigate the difficulties underlying the choice of the number of blank cards.

From a methodological point of view, in our opinion, the application we proposed shows that:

- The common utility scale and the numerical values for the capacities have to be built using user-friendly and easily understandable procedures, which allow decision-makers to clearly comprehend the problem and actively participate in the coconstruction of the evaluation model. In fact, the conclusions stated previously show that the rankings are strongly dependent on these two points.

- The procedures, based on the deck of cards method, proposed in this paper were confirmed as a valid instrument to discuss with the experts and to collect reliable information for building the evaluation model.

- It is important to explore the consequences of the experts' hesitations in the construction of the common utility scale and the capacities of the Choquet integral, because this can provide useful insights into the stability of the rankings.

- From the preceding point, we must conclude that the way the utility values obtained led to ranking the actions in a complete pre-order may not be significant. The way of modeling hesitation and ill-determination through the use of indifference and preference thresholds led to ranking the actions in terms of pseudo orders, which make the results of the Choquet integral more robust. To the best of our knowledge, this is the first time this has been done in a multiple criteria application of the Choquet integral.

As anticipated in the introduction, we can conclude this paper with a descriptive comparison of our method with MACBETH with reference to the construction of utility functions and capacities (Grabisch, 2016; Grabisch \& Labreuche, 2016). It should be remarked, however, that the deck of cards method was built because there was a favourable situation in which the experts wished to work with the deck of cards. This is an alternative method, a dif- ferent option, and it is neither better nor worse than MAсвETH, in general. Comparing these methods in the same contexts would open a new interesting research direction for the scientific community.

In the MAсвETH method the decision-maker is asked to compare pairs of elements. In case of an application to the Choquet integral the elements to be compared are levels for performances on the considered criteria or reference actions to define the capacity. The considered elements are compared through the use of semantic categories: "null", "very weak", "weak", "moderate", "strong", "very strong", and "extreme" (see Bana e Costa De Corte, \& Vansnick, 2016). On the basis of this information, the utility/value scales of the criteria performances and the ratio scale for the capacities of the Choquet integral are determined by solving a specific linear programming problem that allows to represent the qualitative comparisons/judgments on a numerical scale of evaluations. If we compare the МАсBETH approach with the deck of cards method with respect to the elicitation of preferences for the Choquet integral, the following observations can be made (these are the main aspects which, according to our experience, would be favourable to the use of the deck of cards method):

1. In general, МАсветн may require more preference information than the deck of cards method: indeed if there are $n$ elements, MACBETH requires a number of pairwise comparisons ranging between a maximum of $n(n-1) / 2$ (when all pairwise comparisons are made) and $n-1$ (when the minimal number of judgments is considered) ( Bana e Costa De Corte \& Vansnick, 2012 ). The deck of cards method asks to define only the num- ber of blank cards between at most $n$ equivalence classes of elements, that is, at most $n-1$ pieces of information, plus the ratio between the evaluation of the best and the worst classes.

2. The deck of cards method permits to supply a finer assessment of the difference between evaluations of two elements, because the number of blank cards is not a fixed and limited number defined a priori, while, instead this is the case of the semantic categories of MACBETH.

3. The deck of cards method is supporting the intuition of the decision-maker by a simple and understandable visualization given by the cards, while MACBETH, makes use of a more abstract representation for the decision-maker.

4. The deck of cards method permits to easily discuss the values supplied by the method, because the different evaluations assigned to two elements are proportional to the number of cards between them and thus, by changing these cards, the diver- gence between the obtained values and the perception that the decision-maker has of the values can be easily accommodated. In MACBETH the value assigned to each element depends on the whole set of the pairwise comparisons, so that it becomes more difficult to see what has to be modified in order to accommo- date the obtained values to the values perceived and desired by the decision-maker.

5. The deck of cards method permits to take into account robustness concerns with a relatively easy procedure: indeed, it is possible to make a "perturbation" in the number of cards between each pair of elements and then see which are the differences.

In conclusion, we believe that in situations like the one considered in this paper, the deck of cards method is useful because it decreases the cognitive effort of the decision-maker and gives an intuitive support to handle the robustness concerns. There can be other situations where MACBETH will be more adequate. 


\section{Appendix A}

Table 5

Utility values for each action according to criteria $g_{2}, g_{3}, g_{5}$, and $g_{6}$.

\begin{tabular}{|c|c|c|c|c|c|c|c|c|c|c|c|c|}
\hline \multirow[t]{2}{*}{ Actions $\left(a_{i}\right)$} & \multicolumn{3}{|c|}{ Utility $u_{2}\left(a_{i}\right)$} & \multicolumn{3}{|c|}{ Utility $u_{3}\left(a_{i}\right)$} & \multicolumn{3}{|c|}{ Utility $u_{5}\left(a_{i}\right)$} & \multicolumn{3}{|c|}{ Utility $u_{6}\left(a_{i}\right)$} \\
\hline & $\mathrm{b} 1$ & $\mathrm{~b} 2$ & b3 & b1 & $\mathrm{b} 2$ & b3 & b1 & $\mathrm{b} 2$ & b3 & b1 & $\mathrm{b} 2$ & b3 \\
\hline$a_{1}$ & 0.3333 & 0.3889 & 0.2778 & 0.0000 & 0.0000 & 0.0000 & 0.0000 & 0.0000 & 0.0000 & 1.0000 & 1.0000 & 1.0000 \\
\hline$a_{2}$ & 0.3333 & 0.3889 & 0.2778 & 0.6667 & 0.6111 & 0.7222 & 0.6667 & 0.6111 & 0.7222 & 1.0000 & 1.0000 & 1.0000 \\
\hline$a_{3}$ & 0.0000 & 0.0000 & 0.0000 & 0.8333 & 0.7778 & 0.8333 & 0.0000 & 0.0000 & 0.0000 & 1.0000 & 1.0000 & 1.0000 \\
\hline$a_{4}$ & 0.0000 & 0.0000 & 0.0000 & 0.0000 & 0.0000 & 0.0000 & 0.8333 & 0.7778 & 0.8333 & 1.0000 & 1.0000 & 1.0000 \\
\hline$a_{5}$ & 1.0000 & 1.0000 & 1.0000 & 0.0000 & 0.0000 & 0.0000 & 0.3333 & 0.3889 & 0.2778 & 0.0000 & 0.0000 & 0.0000 \\
\hline
\end{tabular}

Table 6

Utility values for each action according to criteria $g_{1}$ and $g_{4}$.

\begin{tabular}{|c|c|c|c|c|c|c|}
\hline \multirow[t]{2}{*}{ Actions $\left(a_{i}\right)$} & \multicolumn{3}{|c|}{ utility $u_{1}\left(a_{i}\right)$} & \multicolumn{3}{|c|}{ utility $u_{4}\left(a_{i}\right)$} \\
\hline & $\overline{c 1}$ & $\mathrm{c} 2$ & c3 & $\mathrm{s} 1$ & s2 & s3 \\
\hline$a_{1}$ & 1.0000 & 0.9922 & 0.9825 & 0.2500 & 0.3077 & 0.3750 \\
\hline$a_{2}$ & 0.9828 & 0.9791 & 0.9535 & 1.0000 & 0.7692 & 0.7500 \\
\hline$a_{3}$ & 0.9310 & 0.9399 & 0.8665 & 0.5500 & 0.4923 & 0.5250 \\
\hline$a_{4}$ & 0.8966 & 0.9138 & 0.8080 & 0.6250 & 0.5385 & 0.5625 \\
\hline$a_{5}$ & 0.0000 & 0.2350 & 0.1825 & 0.0000 & 0.1538 & 0.2500 \\
\hline
\end{tabular}

Table 7

Capacities of the Choquet integral by expert and per option.

\begin{tabular}{|c|c|c|c|c|c|c|c|c|c|c|}
\hline & & \multirow[t]{2}{*}{$z$} & \multicolumn{8}{|c|}{ Capacities of the Choquet Integral } \\
\hline & & & $\mu_{1}$ & $\mu_{2}$ & $\mu_{3}$ & $\mu_{4}$ & $\mu_{5}$ & $\mu_{6}$ & $\mu_{45}$ & $\mu_{15}$ \\
\hline \multirow[t]{4}{*}{ Expert e1 } & c1s1 & 80 & 0.0633 & 0.1782 & 0.1782 & 0.2356 & 0.1207 & 0.0058 & 0.2931 & 0.4655 \\
\hline & $\mathrm{c} 1 \mathrm{~s} 2$ & 80 & 0.0633 & 0.1782 & 0.1782 & 0.2356 & 0.1207 & 0.0058 & 0.2931 & 0.4655 \\
\hline & $\mathrm{c} 2 \mathrm{~s} 1$ & 100 & 0.0567 & 0.1598 & 0.1598 & 0.2113 & 0.1083 & 0.0052 & 0.2629 & 0.5206 \\
\hline & $\mathrm{c} 2 \mathrm{~s} 2$ & 100 & 0.0567 & 0.1598 & 0.1598 & 0.2113 & 0.1083 & 0.0052 & 0.2629 & 0.5206 \\
\hline \multirow[t]{4}{*}{ Expert e2 } & c1s1 & 70 & 0.2987 & 0.3960 & 0.0715 & 0.0715 & 0.0715 & 0.0066 & 0.1364 & 0.4610 \\
\hline & $\mathrm{c} 1 \mathrm{~s} 2$ & 70 & 0.2987 & 0.3960 & 0.0715 & 0.0715 & 0.0715 & 0.0066 & 0.1364 & 0.4610 \\
\hline & $\mathrm{c} 2 \mathrm{~s} 1$ & 70 & 0.2929 & 0.4075 & 0.0639 & 0.0639 & 0.0639 & 0.0066 & 0.1212 & 0.4647 \\
\hline & $c 2 s 2$ & 70 & 0.2643 & 0.4075 & 0.0639 & 0.0639 & 0.0639 & 0.0066 & 0.1212 & 0.4647 \\
\hline \multirow[t]{8}{*}{ Expert e3 } & $\mathrm{c} 1 \mathrm{~s} 1$ & 20 & 0.1512 & 0.0974 & 0.2949 & 0.2051 & 0.3488 & 0.0255 & 0.4206 & 0.5104 \\
\hline & c1s1 & 25 & 0.1491 & 0.0940 & 0.2959 & 0.2041 & 0.3509 & 0.0206 & 0.4243 & 0.5161 \\
\hline & $\mathrm{c} 1 \mathrm{~s} 2$ & 15 & 0.1542 & 0.1020 & 0.2935 & 0.2239 & 0.3458 & 0.0323 & 0.4328 & 0.4851 \\
\hline & $\mathrm{c} 1 \mathrm{~s} 2$ & 20 & 0.1509 & 0.0968 & 0.2951 & 0.2230 & 0.3491 & 0.0247 & 0.4393 & 0.4934 \\
\hline & $\mathrm{c} 2 \mathrm{~s} 1$ & 20 & 0.1665 & 0.0962 & 0.2895 & 0.2192 & 0.3423 & 0.0259 & 0.4126 & 0.5180 \\
\hline & $\mathrm{c} 2 \mathrm{~s} 1$ & 25 & 0.1647 & 0.0928 & 0.2904 & 0.2186 & 0.3443 & 0.0210 & 0.4162 & 0.5240 \\
\hline & $\mathrm{c} 2 \mathrm{~s} 2$ & 20 & 0.1585 & 0.0919 & 0.2750 & 0.2250 & 0.3249 & 0.0254 & 0.4247 & 0.5079 \\
\hline & $c 2 s 2$ & 30 & 0.1552 & 0.0862 & 0.2759 & 0.2241 & 0.3276 & 0.0172 & 0.4310 & 0.5172 \\
\hline \multirow[t]{4}{*}{ Expert e4 } & $\mathrm{c} 1 \mathrm{~s} 1$ & 90 & 0.2091 & 0.3114 & 0.0864 & 0.1273 & 0.1273 & 0.0046 & 0.3114 & 0.4136 \\
\hline & $\mathrm{c} 1 \mathrm{~s} 2$ & 90 & 0.1225 & 0.2990 & 0.1814 & 0.2402 & 0.2402 & 0.0049 & 0.3186 & 0.4363 \\
\hline & $\mathrm{c} 2 \mathrm{~s} 1$ & 90 & 0.4397 & 0.2771 & 0.0603 & 0.1145 & 0.1145 & 0.0061 & 0.2229 & 0.5481 \\
\hline & $c 2 s 2$ & 100 & 0.1223 & 0.3188 & 0.1616 & 0.2402 & 0.2402 & 0.0044 & 0.3188 & 0.4367 \\
\hline
\end{tabular}


Table 8

Distribution of the utility values $\min ^{z}\left\{a_{i}\right\}$.

\begin{tabular}{|c|c|c|c|c|c|c|c|c|}
\hline$\Delta^{z}\left(a_{i}\right)$ & $N b$. & $\min ^{z}\left\{a_{i}\right\}$ & $\Delta^{z}\left(a_{i}\right)$ & $N b$. & $\min ^{z}\left\{a_{i}\right\}$ & $\Delta^{z}\left(a_{i}\right)$ & $N b$. & $\min ^{z}\left\{a_{i}\right\}$ \\
\hline 0.000017 & 1 & 0.624699 & 0.000596 & 1 & 0.219691 & 0.002475 & 1 & 0.218640 \\
\hline 0.000077 & 1 & 0.490508 & 0.000597 & 1 & 0.663873 & 0.002509 & 1 & 0.179804 \\
\hline 0.000080 & 1 & 0.450089 & 0.000608 & 1 & 0.654536 & 0.002517 & 1 & 0.180248 \\
\hline 0.000088 & 2 & 0.316572 & 0.000611 & 2 & $0.739096,0.476550$ & 0.002589 & 1 & 0.199511 \\
\hline 0.000099 & 1 & 0.444453 & 0.000612 & 1 & 0.654743 & 0.002602 & 1 & 0.211009 \\
\hline 0.000104 & 1 & 0.443163 & 0.000635 & 1 & 0.535318 & 0.002713 & 1 & 0.255884 \\
\hline 0.000109 & 1 & 0.445917 & 0.000638 & 1 & 0.454520 & 0.002720 & 1 & 0.191514 \\
\hline 0.000109 & 2 & $0.418322(2)$ & 0.000663 & 1 & 0.477044 & 0.002721 & 1 & 0.295259 \\
\hline 0.000110 & 1 & 0.441044 & 0.000670 & 1 & 0.531768 & 0.002730 & 1 & 0.295765 \\
\hline 0.000113 & 3 & $0.404508,0.420436(2)$ & 0.000677 & 1 & 0.505307 & 0.002863 & 1 & 0.237685 \\
\hline 0.000114 & 1 & 0.391164 & 0.000677 & 2 & $0.505307(2)$ & 0.006217 & 1 & 0.540023 \\
\hline 0.000115 & 1 & 0.377820 & 0.000683 & 1 & 0.323549 & 0.006266 & 1 & 0.523888 \\
\hline 0.000136 & 1 & 0.433587 & 0.000690 & 1 & 0.305839 & 0.006536 & 1 & 0.647971 \\
\hline 0.000143 & 1 & 0.400855 & 0.000692 & 1 & 0.454378 & 0.006600 & 2 & $0.518273(2)$ \\
\hline 0.000147 & 1 & 0.434745 & 0.000700 & 1 & 0.316864 & 0.006608 & 1 & 0.375475 \\
\hline 0.000148 & 2 & $0.402968,0.505424$ & 0.000703 & 1 & 0.505984 & 0.007937 & 1 & 0.278661 \\
\hline 0.000242 & 1 & 0.470206 & 0.000706 & 1 & 0.300246 & 0.008125 & 1 & 0.283817 \\
\hline 0.000243 & 2 & $0.456862,0.633636$ & 0.000711 & 2 & $0.781706,0.283628$ & 0.008189 & 1 & 0.246878 \\
\hline 0.000253 & 1 & 0.526572 & 0.000760 & 1 & 0.446246 & 0.008374 & 1 & 0.252102 \\
\hline 0.000256 & 1 & 0.423853 & 0.000789 & 1 & 0.423580 & 0.010966 & 2 & $0.503325(2)$ \\
\hline 0.000271 & 1 & 0.525573 & 0.000828 & 1 & 0.377396 & 0.013321 & 1 & 0.264110 \\
\hline 0.000274 & 1 & 0.631695 & 0.000836 & 2 & $0.387464(2)$ & 0.013587 & 1 & 0.443518 \\
\hline 0.000286 & 1 & 0.624699 & 0.000860 & 1 & 0.363202 & 0.013640 & 1 & 0.268899 \\
\hline 0.000289 & 1 & 0.419881 & 0.000870 & 2 & $0.372178(2)$ & 0.013669 & 1 & 0.420170 \\
\hline 0.000304 & 1 & 0.629755 & 0.001133 & 1 & 0.525514 & 0.014552 & 1 & 0.435537 \\
\hline 0.000312 & 1 & 0.624942 & 0.001169 & 1 & 0.717182 & 0.017012 & 1 & 0.288827 \\
\hline 0.000317 & 1 & 0.441128 & 0.001331 & 2 & $0.515140(2)$ & 0.026766 & 1 & 0.454520 \\
\hline 0.000323 & 1 & 0.429867 & 0.001454 & 1 & 0.377993 & & & \\
\hline 0.000352 & 1 & 0.437578 & 0.001456 & 1 & 0.378144 & & & \\
\hline 0.000377 & 1 & 0.739970 & 0.001483 & 1 & 0.359058 & & & \\
\hline 0.000418 & 1 & 0.244524 & 0.001489 & 1 & 0.382083 & & & \\
\hline 0.000444 & 1 & 0.638146 & 0.001518 & 1 & 0.362573 & & & \\
\hline 0.000469 & 1 & 0.631739 & 0.001524 & 1 & 0.757368 & & & \\
\hline 0.000483 & 1 & 0.641638 & 0.001573 & 1 & 0.334668 & & & \\
\hline 0.000500 & 1 & 0.621695 & 0.001608 & 1 & 0.338183 & & & \\
\hline 0.000519 & 1 & 0.629998 & 0.001935 & 1 & 0.715150 & & & \\
\hline 0.000543 & 1 & 0.217881 & 0.002166 & 1 & 0.185228 & & & \\
\hline 0.000557 & 1 & 0.652823 & 0.002169 & 1 & 0.198819 & & & \\
\hline 0.000573 & 1 & 0.707185 & 0.002291 & 1 & 0.326927 & & & \\
\hline 0.000582 & 1 & 0.654507 & 0.002295 & 1 & 0.340517 & & & \\
\hline
\end{tabular}


Table 9

Distribution of the utility values $\min ^{b c}\left\{a_{i}\right\}$.

\begin{tabular}{|c|c|c|c|c|c|}
\hline$\Delta^{b c}\left(a_{i}\right)$ & $N b$. & $\min ^{b c}\left\{a_{i}\right\}$ & $\Delta^{b c}\left(a_{i}\right)$ & $\mathrm{Nb}$ & $\min ^{b c}\left\{a_{i}\right\}$ \\
\hline 0.0000 & 1 & 0.237685 & 0.0066 & 2 & $0.647956,0.447756$ \\
\hline 0.0001 & 4 & $0.278661,0.739970,0.283817,0.739096$ & 0.0067 & 1 & 0.3754 \\
\hline 0.0002 & 1 & 0.717182 & 0.0068 & 2 & $0.518273(2)$ \\
\hline 0.0003 & 3 & $0.444453,0.445991,0.715150$ & 0.0070 & 1 & 0.41706 \\
\hline 0.0004 & 3 & $0.423580,0.505545(2)$ & 0.0073 & 2 & $0.469786,0.434129$ \\
\hline 0.0005 & 2 & $0.433587,0.255884$ & 0.0074 & 1 & 0.41277 \\
\hline 0.0006 & 2 & $0.441044,0.443163$ & 0.0077 & 1 & 0.430190 \\
\hline 0.0007 & 1 & 0.191514 & 0.0079 & 1 & 0.420170 \\
\hline 0.0009 & 1 & 0.450089 & 0.0084 & 1 & 0.450207 \\
\hline 0.0010 & 1 & 0.211009 & 0.0088 & 1 & 0.445752 \\
\hline 0.0011 & 2 & $0.651679,0.445917$ & 0.0093 & 1 & $0.316572,0.467253$ \\
\hline 0.0013 & 1 & 0.199511 & 0.0100 & 1 & 0.525954 \\
\hline 0.0014 & 1 & 0.434745 & 0.0102 & 1 & 0.185228 \\
\hline 0.0015 & 1 & 0.638146 & 0.0104 & 1 & 0.522015 \\
\hline 0.0016 & 2 & $0.218640,0.632009$ & 0.0111 & 1 & 0.198819 \\
\hline 0.0017 & 4 & $0.378144,0.377993,0.264110,0.268899$ & 0.0114 & 1 & 0.334668 \\
\hline 0.0018 & 3 & $0.641638,0.504873,0.457105$ & 0.0116 & 1 & 0.445456 \\
\hline 0.0019 & 5 & $0.652823,0.652595,0.663873,0.524762,0.470448$ & 0.0119 & 1 & 0.338183 \\
\hline 0.0021 & 1 & 0.382083 & 0.0130 & 1 & 0.270598 \\
\hline 0.0022 & 1 & 0.707185 & 0.0131 & 1 & 0.217881 \\
\hline 0.0025 & 1 & 0.375413 & 0.0132 & 1 & 0.287046 \\
\hline 0.0026 & 1 & 0.781706 & 0.0134 & 1 & 0.303494 \\
\hline 0.0028 & 1 & 0.388449 & 0.0146 & 1 & 0.435503 \\
\hline 0.0029 & 4 & $0.523888,0.628838,0.621844,0.622084$ & 0.0148 & 1 & 0.244524 \\
\hline 0.0030 & 6 & $0.654507,0.631739,0.757368,0.540023,0.513441(2)$ & 0.0172 & 1 & 0.454520 \\
\hline 0.0031 & 2 & $0.401485,0.621604$ & 0.0185 & 1 & 0.179804 \\
\hline 0.0039 & 4 & $0.246878,0.252102,0.416507(2)$ & 0.0186 & 1 & 0.180248 \\
\hline 0.0041 & 3 & $0.625667,0.400855,0.398896$ & 0.0187 & 1 & 0.358703 \\
\hline 0.0044 & 1 & 0.629998 & 0.0189 & 1 & 0.326927 \\
\hline 0.0046 & 4 & $0.418322(2), 0.367536(2)$ & 0.0191 & 2 & $0.368327(2)$ \\
\hline 0.0047 & 1 & 0.358490 & 0.0197 & 1 & 0.340517 \\
\hline 0.0049 & 1 & 0.359058 & 0.0201 & 1 & 0.485521 \\
\hline 0.0052 & 1 & 0.621695 & 0.0210 & 1 & 0.469577 \\
\hline 0.0053 & 1 & 0.362573 & 0.0234 & 1 & 0.282435 \\
\hline 0.0054 & 1 & 0.318138 & 0.0248 & 1 & 0.219691 \\
\hline 0.0056 & 1 & 0.300287 & 0.0317 & 2 & $0.295259,0.295765$ \\
\hline 0.0062 & 2 & $0.503325(2)$ & 0.0352 & 1 & 0.491664 \\
\hline 0.0064 & 1 & 0.423769 & 0.0353 & 1 & 0.490585 \\
\hline
\end{tabular}

Table 10

Configuration by expert for bh and zmin.

\begin{tabular}{|c|c|c|c|c|}
\hline \multicolumn{3}{|c|}{ Expert Configuration Pseudo order } & \multicolumn{2}{|c|}{ Configuration Pseudo order } \\
\hline e1 & $\begin{array}{l}\text { c1s1onc } \\
\text { c1s1ace } \\
\text { c1s2onc } \\
\text { c1s2ace }\end{array}$ & $\begin{array}{l}a_{2} P a_{4} P a_{3} P a_{5} P a_{1} \\
a_{2} P a_{4} P a_{3} P a_{5} P a_{1} \\
a_{2} P a_{4} P a_{3} P a_{5} P a_{1} \\
a_{2} P a_{4} P a_{3} P a_{5} P a_{1}\end{array}$ & $\begin{array}{l}\text { c2s1onc } \\
\text { c2s1ace } \\
\text { c2s2onc } \\
\text { c2s2ace }\end{array}$ & $\begin{array}{l}a_{2} \mathrm{~Pa}_{4} \mathrm{~Pa}_{3} \mathrm{~Pa}_{5} \mathrm{~Pa}_{1} \\
a_{2} \mathrm{~Pa}_{4} \mathrm{~Pa}_{3} \mathrm{~Pa}_{5} \mathrm{~Pa}_{1} \\
a_{2} \mathrm{~Pa}_{4} \mathrm{~Pa}_{5} \mathrm{Qa}_{3} P a_{1} \\
a_{2} \mathrm{~Pa}_{4} P\left(a_{3} I a_{5}\right) \mathrm{Pa}_{1}\end{array}$ \\
\hline e2 & $\begin{array}{l}\text { c1s1onc } \\
\text { c1s1ace } \\
\text { c1s2onc } \\
\text { c1s2ace }\end{array}$ & $\begin{array}{l}a_{2} \mathrm{~Pa}_{1} \mathrm{~Pa}_{4} \mathrm{Qa}_{5} \mathrm{~Pa}_{3} \\
a_{2} \mathrm{~Pa}_{1} \mathrm{~Pa}_{4} \mathrm{~Pa}_{5} \mathrm{~Pa}_{3} \\
a_{2} \mathrm{~Pa}_{1} P\left(a_{4} I a_{5}\right) \mathrm{Pa}_{3} \\
a_{2} P a_{1} P\left(a_{4} I a_{5}\right) P a_{3}\end{array}$ & $\begin{array}{l}\text { c2s1onc } \\
\text { c2s1ace } \\
\text { c2s2onc } \\
\text { c2s2ace }\end{array}$ & $\begin{array}{l}a_{2} \mathrm{~Pa}_{1} \mathrm{~Pa}_{5} \mathrm{Qa}_{4} P a_{3} \\
a_{2} \mathrm{~Pa}_{1} P\left(a_{4} I a_{5}\right) \mathrm{Pa}_{3} \\
a_{2} \mathrm{~Pa}_{5} \mathrm{~Pa}_{1} \mathrm{Qa}_{4} P a_{3} \\
a_{2} \mathrm{~Pa}_{5} \mathrm{~Pa}_{1} \mathrm{Qa}_{4} P a_{3}\end{array}$ \\
\hline e3 & $\begin{array}{l}\text { c1s1onc } \\
\text { c1s1ace } \\
\text { c1s2onc } \\
\text { c1s2ace }\end{array}$ & $\begin{array}{l}a_{2} \mathrm{~Pa}_{3} \mathrm{Qa}_{4} \mathrm{~Pa}_{1} \mathrm{~Pa}_{5} \\
a_{2} \mathrm{~Pa}_{3} \mathrm{~Pa}_{4} \mathrm{~Pa}_{1} \mathrm{~Pa}_{5} \\
\text { mutual-strengthening } \\
\text { mutual-strengthening }\end{array}$ & $\begin{array}{l}\text { c2s1onc } \\
\text { c2s1ace } \\
\text { c2s2onc } \\
\text { c2s2ace }\end{array}$ & $\begin{array}{l}a_{2} \mathrm{~Pa}_{3} \mathrm{~Pa}_{4} \mathrm{~Pa}_{1} \mathrm{~Pa}_{5} \\
a_{2} \mathrm{~Pa}_{3} \mathrm{~Pa}_{4} \mathrm{~Pa}_{1} \mathrm{~Pa}_{5} \\
a_{2} P\left(a_{4} I a_{3}\right) \mathrm{Pa}_{1} \mathrm{~Pa}_{5} \\
a_{2} \mathrm{~Pa}_{3} \mathrm{Qa}_{4} \mathrm{~Pa}_{1} P a_{5}\end{array}$ \\
\hline e4 & $\begin{array}{l}\text { c1s1onc } \\
\text { c1s1ace } \\
\text { c1s2onc } \\
\text { c1s2ace }\end{array}$ & $\begin{array}{l}\text { mutual-weakening } \\
\text { mutual-weakening } \\
a_{2} P a_{4} P\left(a_{5} I a_{3}\right) P a_{1} \\
a_{2} P a_{4} P a_{5} Q a_{3} P a_{1}\end{array}$ & $\begin{array}{l}\text { c2s1onc } \\
\text { c2s1ace } \\
\text { c2s2onc } \\
\text { c2s2ace }\end{array}$ & $\begin{array}{l}\text { mutual-strengthening } \\
\text { mutual-strengthening } \\
a_{2} \mathrm{~Pa}_{5} \mathrm{~Pa}_{4} \mathrm{~Pa}_{3} \mathrm{~Pa}_{1} \\
a_{2} \mathrm{~Pa}_{5} \mathrm{~Pa}_{4} \mathrm{~Pa}_{3} \mathrm{~Pa}_{1}\end{array}$ \\
\hline
\end{tabular}

Table 11

Pseudo order by expert for bh and zmin.

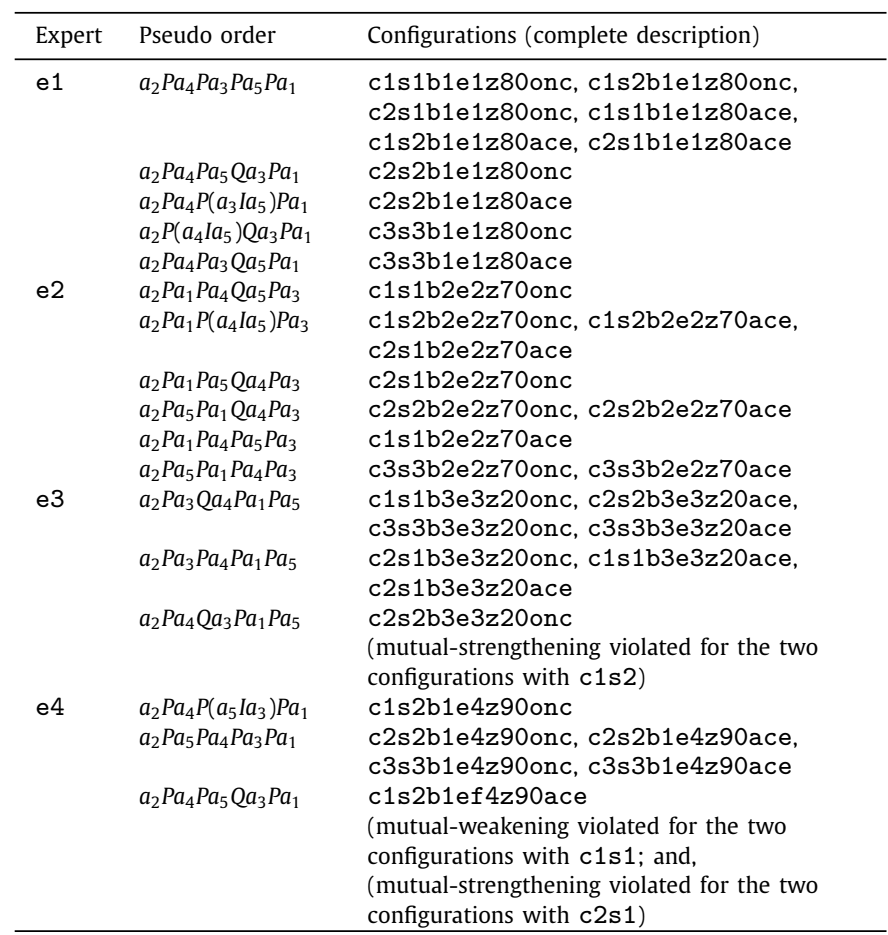




\section{Appendix C}

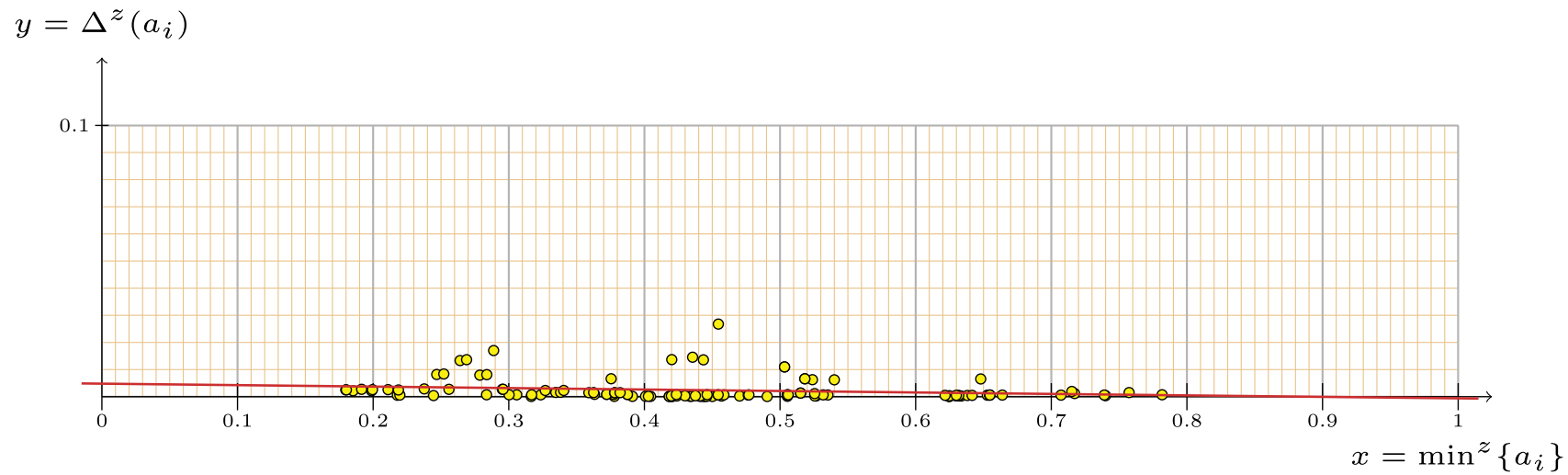

Fig. 1. Regression line for the points $\left(\min ^{z}\left\{a_{i}\right\}, \Delta^{z}\left(a_{i}\right)\right): y=-0.0054 x+0.0048$.

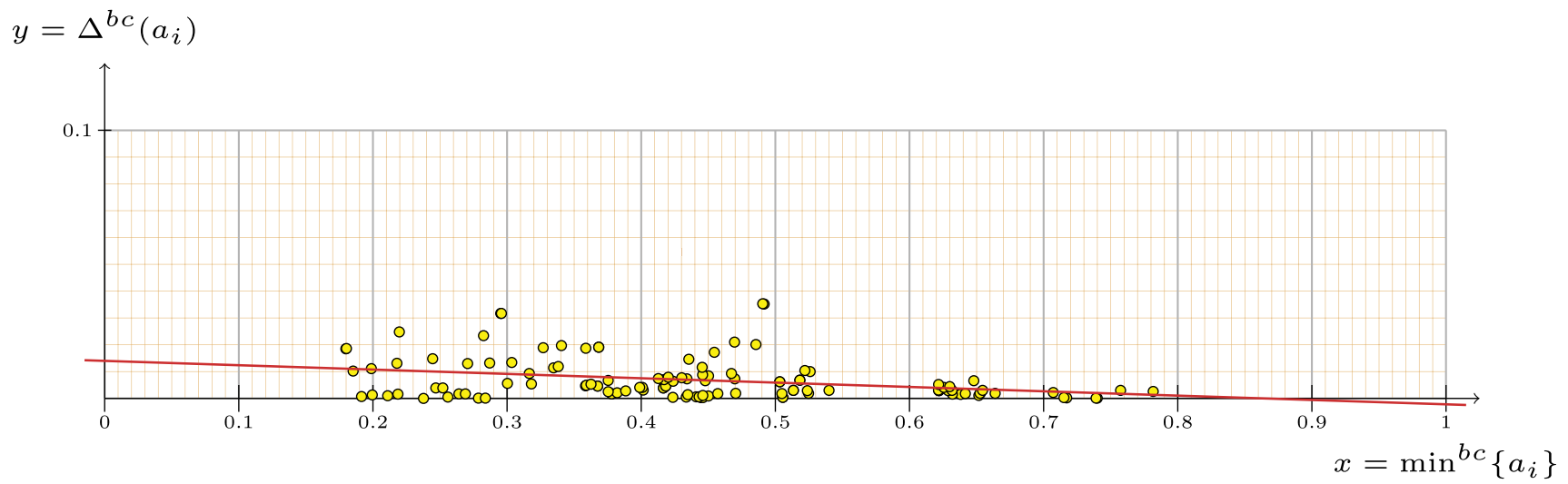

Fig. 2. Regression line for the points $\left(\min ^{b c}\left\{a_{i}\right\}, \Delta\left(a_{i}\right)\right): y=-0.0162 x+0.014$.

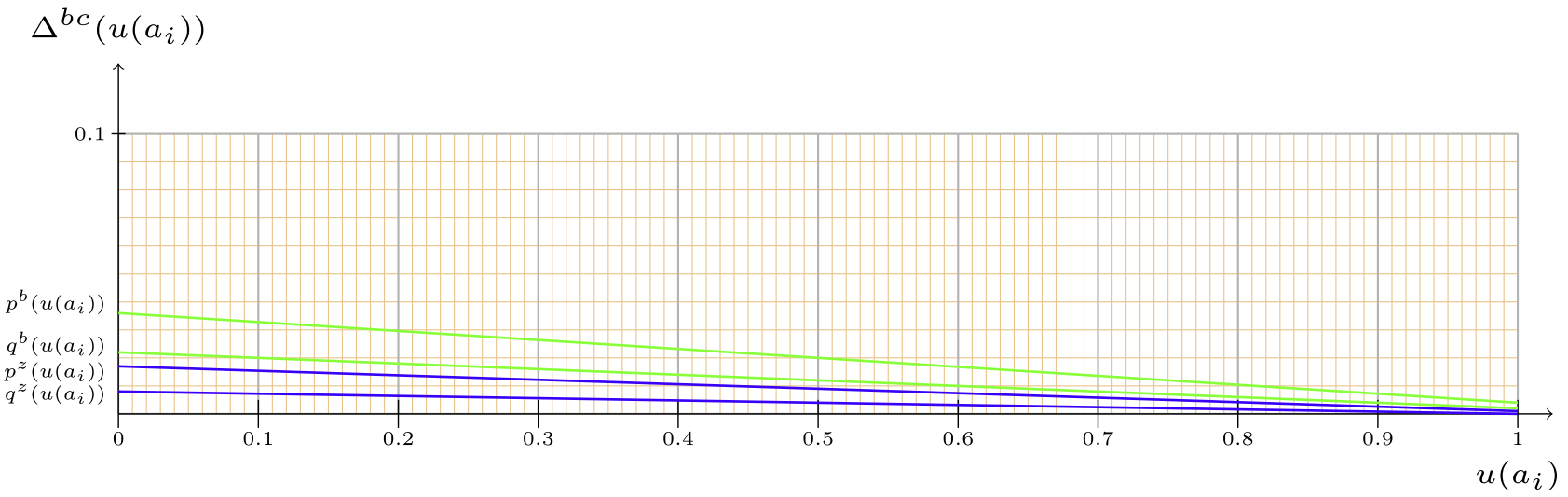

Fig. 3. Indifference and preference thresholds lines. 


\section{References}

Angilella, S., Corrente, S., \& Greco, S. (2015). Stochastic multiobjective acceptability analysis for the Choquet integral preference model and the scale construction problem. European Journal of Operational Research, 240, 172-182.

Angilella, S., Greco, S., Lamantia, F., \& Matarazzo, B. (2004). Assessing non-additive utility for multicriteria decision aid. European Journal of Operational Research, $158,734-744$

Angilella, S., Greco, S., \& Matarazzo, B. (2010). Non-additive robust ordinal regression: A multiple criteria decision model based on the Choquet integral. European Journal of Operational Research, 201, 277-288.

Bana e Costa, C., De Corte, J.-M., \& Vansnick, J.-C. (2012). MAcBETH. International Journal of Information Technology \& Decision Making, 11, 359-387.

Bana e Costa, C., De Corte, J.-M., \& Vansnick, J.-C. (2016). On the mathematical foundations of MAсвETH. In S. Greco, M. Ehrgott, \& J.-R. Figueira (Eds.), Multiple criteria decision analysis: State of the art surveys (pp. 421-463). New York, NY, USA: Springer Science + Business Media Inc.

Boerboom, L., \& Ferretti, V. (2014). Actor network theory perspective on a forestry decision support system design. Scandinavian Journal of Forest Research, 29, 84-95.

Bottero, M., Ferretti, V., Figueira, J.-R., Greco, S., \& Roy, B. (2015). Dealing with a multiple criteria environmental problem with interaction effects between criteria through an extension of the ELECTRE III method. European Journal of Operational Research, 245, 837-850.

Bottero, M., Ferretti, V., \& Pomarico, S. (2014). Assessing different possibilities for the reuse of an open-pit quarry using the Choquet integral. Journal of Multi-Criteria Decision Analysis, 21, 25-41.

Choquet, G. (1953). Theory of capacities. Annales de l'Institut Fourier, 5, 131-295.

Dente, B. (2014). Understanding Policy Decisions. Milan, Italy: PoliMI SpringerBriefs.

Ferretti, V. (2016). From stakeholders analysis to cognitive mapping and multi-attribute value theory: An integrated approach for policy support. European Journal of Operational Research, 253, 524-541.

Figueira, J., \& Roy, B. (2002). Determining the weights of criteria in the Electre type methods with a revised Simos' procedure. European Journal of Operational Research, 139, 317-326.

Figueira, J.-R., Greco, S., \& Roy, B. (2009). ElECTRE methods with interaction between criteria: An extension of the concordance index. European Journal of Operational Research, 199, 478-495.

Fujimoto, K. (2010). Representations of importance and interaction of fuzzy measures, capacities, games and its extensions: A survey. In V.-N. Huynh, Y. Nakamori, J. Lawry, \& M. Inuiguchi (Eds.), Integrated uncertainty management and applications (pp. 115-126). Berlin, Germany: Springer-Verlag.

Fujimoto, K., Kojadinovic, I., \& Marichal, J.-L. (2006). Axiomatic characterizations of probabilistic and cardinal-probabilistic interaction indices. Games and Economic Behavior, 55, 72-99.

Grabisch, M. (1996a). The application of fuzzy integrals in multicriteria decision making. European Journal of Operational Research, 89, 445-456.

Grabisch, M. (1996b). The representation of importance and interaction of features by fuzzy measures. Pattern Recognition Letters, 17, 567-575.

Grabisch, M. (1997). $k$-order additive discrete fuzzy measures and their

representation. Fuzzy Sets and Systems, 92, 167-189.

Grabisch, M. (2016). Set functions, games and capacities in decision making. Berlin, Germany: Springer-Verlag.
Grabisch, M., \& Labreuche, C. (2005). Bi-capacities II: The Choquet integral. Fuzzy Sets and Systems, 151, 237-259.

Grabisch, M., \& Labreuche, C. (2010). A decade of application of the Choquet and Sugeno integrals in multi-criteria decision aid. Annals of Operations Research, $175, \quad 247-286$.

Grabisch, M., \& Labreuche, C. (2016). Fuzzy measures and integrals in MCDA.

In S. Greco, M. Ehrgott, \& J.-R. Figueira (Eds.), Multiple criteria decision analysis: State of the art surveys (pp. 553-603). New York, NY, USA: Springer Science + Business Media Inc.

Grabisch, M., Marichal, J.-L., \& Roubens, M. (2000). Equivalent representations of set functions. Mathematics of Operations Research, 25, 157-178.

Grabisch, M., \& Roubens, M. (1999). An axiomatic approach to the concept of interaction among players in cooperative games. International Journal of Game Theory, 28, 547-565.

Greco, S., \& Rindone, F. (2014). The bipolar Choquet integral representation. Theory and Decision, 77, 1-29.

Kojadinovic, I. (2007a). Minimum variance capacity identification. European

Jourzal Operational Research, 177, 498-514.

Kojadinovic, I. (2007b). A weight-based approach to the measurement of the interaction among criteria in the framework of aggregation by the bipolar Choquet integral. European Journal of Operational Research, 179, 498-517.

Marichal, J.-L., \& Roubens, M. (2000). Determination of weights of interacting criteria from a reference set. European Journal of Operational Research, 124, 641-650.

Meyer, P., \& Roubens, M. (2005). Choice, ranking and sorting in fuzzy multiple criteria decision aid. In J.-R. Figueira, S. Greco, \& M. Ehrgott (Eds.), Multiple criteria decision analysis: State of the art surveys (pp. 471-506). New York, NY, USA: Springer Science + Business Media Inc.

Morgan, D. (1988). Focus group as qualitative research. Beverly Hills, CA, USA: Sage Publications.

Mori, T., \& Murofushi, T. (1989). An analysis of evaluation model using fuzzy measure and the Choquet integral. In Proceedings of the 5-th fuzzy system symposium, Kobe, Japan (pp. 207-212)

Murofushi, T., \& Soneda, S. (1993). Techniques for reading fuzzy measures (III): Interaction index. In Proceedings of the 9-th fuzzy system symposium, Sapporo, Japan (pp. 693-696).

Pictet, J., \& Bollinger, D. (2008). Extended use of the cards procedure as a simple elicitation technique for MAVT. application to public procurement in switzerland. European Journal of Operational Research, 185, 1300-1307.

Roy, B., Figueira, J.-R., \& Almeida-Dias, J. (2014). Discriminating thresholds as a too to cope with imperfect knowledge in multiple criteria decision aiding: Theoretical results and practical issues. OMEGA, The International Journal of Management Science, 43, 9-20.

Roy, B., \& Vincke, P. (1984). Relational systems of preference with one or morpseudo-criteria: Some new concepts and results. Management Science, 30, 1323-1335.

Siskos, E., \& Tsotsolas, N. (2015). Elicitation of criteria importance weights through the Simos method: A robustness concern. European Journal of Operational Research, 246, 543-553.

Stewart, D., \& Shamdasani, P. (1990). Focus groups: theory and practice. Newbury Park, CA, USA: Sage Publications.

(ew von Winterfeldt, D., \& Edwards, W. (1986). Decision analysis research.Cambridge, England: Cambridge University Press. 